\title{
Numerical analysis of Urea-SCR sprays under cross-flow conditions
}

by

\author{
Jakob Heide
}





\title{
KUNGLIGA TEKNISKA HÖGSKOLAN
}

\author{
Abstract \\ Department of Mechanics \\ Master of Science \\ Numerical analysis of Urea-SCR sprays under cross-flow conditions \\ by Jakob HeIDE \\ The mixing and evaporation of Diesel Exhaust Fluid (DEF) inside an Urea \\ Selective Catalyst Reduction (SCR) chamber has been numerically investi- \\ gated. The first task in this work has been to first look into the numerical \\ framework and assess the models available in a commercial CFD software \\ (ANSYS Fluent 14.5). Secondly the knowledge inherited from the model \\ sensitivity analysis will be applied on the practical case of an Urea-SCR \\ mixing chamber. Mass flow rate and temperature effects of the exhaust \\ gas on the mixing and evaporation of the DEF spray has been investigated. \\ The results indicate that evaporation rates inside the mixing chamber are \\ dependent on the mass flow rate of the exhaust gas but not on the tem- \\ perature due to compressibility effects of the exhaust gas. For a constant \\ mass flow rate an increase in temperature decreases the residence time of \\ droplets (due to compressibility) with a similar order of magnitude as the \\ individual droplet evaporation rate increases (due to higher temperature) \\ thus the two effects balances each other. The results could potentially con- \\ tribute to the development and optimization of current SCR systems.
}

Keywords: Lagrangian Particle Tracking, Selective Catalyst Reduction, droplet evaporation, sub-models. 



\section{Acknowledgements}

First and foremost, I have to thank my research supervisor, PhD Mireia Altimira. Without your assistance and dedicated involvement in every step throughout the process, this paper would have never been accomplished.

I also want to thank PhD Giovanni Lacagnina for contributing with experimental data and practical know how regarding the experimental equipment used. As the work of this thesis was in collaboration with the research group CCGEx (Competence Center of Gas Exchange) I would in addition like to express my gratitude for their help. 



\section{Contents}

$\begin{array}{ll}\text { Abstract } & \text { iii }\end{array}$

Acknowledgements $\quad$ v

1 Introduction 1

1.1 Technical background $\ldots \ldots \ldots \ldots \ldots$

1.2 Motivation and structure . . . . . . . . . . . . . 1

1.3 Literature review . . . . . . . . . . . . . . . . 3

2 Models and methods 5

2.1 Numerical procedure . . . . . . . . . . . . . . . 5

2.1.1 Governing equations . . . . . . . . . . . 5

2.1.2 Finite volume method . . . . . . . . . . 5

2.1.3 Turbulence model . . . . . . . . . . . . . 6

2.1.4 Boundary layer . . . . . . . . . . . . . . . 7

2.1.5 Lagrangian Particle Tracking . . . . . . . . . . . . . 8

2.2 Experimental procedure . . . . . . . . . . . . . . 15

2.3 Case setup . . . . . . . . . . . . . . . 16

2.3.1 Pre study - Water mist nozzle . . . . . . . . . . . 16

2.3.2 Urea-SCR dosing chamber . . . . . . . . . . . . . . . 19

3 Results and discussion 23

3.1 Sensitivity to sub-models - Water mist nozzle case . . . . . 23

3.1 .1 Turbulence . . . . . . . . . . . . . . . . . . . 24

3.1 .2 Evaporation . . . . . . . . . . . . 25

3.1 .3 Breakup .................. 26

3.1.4 Summary of sensitivity study . . . . . . . . . . . 27

3.2 Urea-SCR dosing chamber . . . . . . . . . . . . . . . . 28

3.2.1 Validation of size distribution . . . . . . . . . 28

3.2 .2 Mass flow rate . . . . . . . . . . . . . . . . 29

3.2 .3 Temperature . . . . . . . . . . . . . . . . 32

3.2 .4 Parcel sensitivity . . . . . . . . . . . . . 34

4 Conclusion $\quad 37$

Bibliography $\quad 39$ 



\section{List of Figures}

1.1 Work flow, ${ }^{1}$ Iannantuoni et al., $2013 \ldots \ldots$. . . . . . . . 2

2.1 The turbulent boundary layer . . . . . . . . . . . . . 8

2.2 Physical phenomena around the liquid film . . . . . . . . 13

2.3 Solution procedure one-way coupled calculations . . . . . . 14

2.4 Solution procedure two-way coupled calculations . . . . . . 15

2.5 Optical arrangement of PDA technique . . . . . . . . . 15

2.6 The dosing chamber unit . . . . . . . . . . . . . . 16

2.7 O-grid mesh . . . . . . . . . . . . . . . . 17

2.8 The dosing chamber unit . . . . . . . . . . . . . . . . . 19

2.9 Mesh of dosing chamber, unstructured mesh with 5 prism layers . . . . . . . . . . . . . . . . 20 20

2.10 Fitted Weibull distribution to experimental data . . . . . . 21

3.1 Droplets coloured by diameter at $\mathrm{t}=0.8 \mathrm{~s}$ after injection start 23

3.2 Number averaged radial size distribution for Discrete Random Walk turbulence coupling vs none (case $1 \& 2$ ) . . . . .

3.3 Number averaged radial velocity distribution for Discrete Random Walk turbulence coupling vs none (case $1 \& 2$ ) . . .

3.4 Number averaged radial size distribution for different evap-

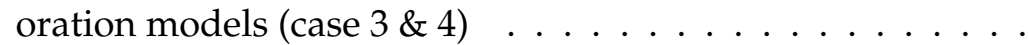

3.5 Number averaged radial velocity distribution for different evaporation models (case $3 \& 4$ ) . . . . . . . . . . . 26

3.6 Number averaged radial size distribution for different breakup models (case $5,6,7 \& 8$ ) . . . . . . . . . . . . . 26

3.7 Number averaged radial velocity distribution for different breakup models (case $5,6,7 \& 8$ ) . . . . . . . . . 27

3.8 Sampling lines at $\mathrm{L}=1 \mathrm{D}, 2 \mathrm{D}$ and $3 \mathrm{D}$ downstream of the injector where $\mathrm{D}$ is the inlet diamater $D=12 \mathrm{~cm} \ldots \ldots .$.

3.9 Probability Density Function for the droplet size distribution (Case 1a) . . . . . . . . . . . . . . . . .

3.10 Velocity magnitude contours for mild cross-flow $(400 \mathrm{~kg} / \mathrm{h})$ (above) and high cross-flow $\left(1400 \mathrm{~kg} / \mathrm{h}\right.$ ) (below), $300^{\circ} \mathrm{C}$ (Case $2 b \& 4 b) \ldots \ldots \ldots \ldots \ldots \ldots$

3.11 Streamlines colored by velocity magnitude for high crossflow, $300{ }^{\circ} \mathrm{C}$ (Case $\left.4 \mathrm{~b}\right)$. . . . . . . . . . . . . . . . .

3.12 Turbulent intensity for mild cross-flow (above) and high crossflow (below) based on mean velocity magnitude calculated as $15.4 \mathrm{~m} / \mathrm{s}$ at the inlet of mild cross-flow case, $300{ }^{\circ} \mathrm{C}$ (Case $2 b \& 4 b) \ldots \ldots \ldots \ldots \ldots \ldots \ldots$

3.13 Vertical diameter distribution at a downstream distance of $1 \mathrm{D}, 2 \mathrm{D}$ and $3 \mathrm{D}$ (Case $2 \mathrm{~b} \& 4 \mathrm{~b}) \ldots \ldots \ldots$

\section{(3)} 4

15

6

9


3.14 Vertical velocity distribution at a downstream distance of 1D, 2D and 3D (Case 2b \& 4b) . . . . . . . . . . . . . 31

3.15 Spray mass flow rate downstream (Case $2 b \& 4 b) \quad \ldots . . .32$

3.16 Vertical diameter distribution at a downstream distance of 1D, 2D and 3D (Case 4a, 4b \& 4c) . . . . . . . . . 33

3.17 Vertical velocity distribution at a downstream distance of 1D, $2 \mathrm{D}$ and $3 \mathrm{D}$ (Case $4 \mathrm{a}, 4 \mathrm{~b} \& 4 \mathrm{c}) \ldots \ldots \ldots$

3.18 Spray mass flow rate downstream (Case $4 a, 4 b \& 4 c) \ldots \ldots$

3.19 Vertical diameter distribution at $x=1 D, 2 D, 3 D$. P200 $=200$ 000 parcels $/ \mathrm{s}$ and $\mathrm{P} 1000=1000000$ parcels $/ \mathrm{s}$ (Case 4b \& 4b_s) 35

3.20 Vertical velocity distribution at $\mathrm{x}=1 \mathrm{D}, 2 \mathrm{D}, 3 \mathrm{D}$. P200 $=200$ 000 parcels /s and P1000 $=1000000$ parcels /s (Case 4b \& 4b_s) 35

3.21 Spray mass flow rate downstream, P200 $=200000$ parcels $/ \mathrm{s}$ and P1000 $=1000000$ parcels $/ \mathrm{s}$ (Case $4 \mathrm{~b} \& 4$ b_s) . . . . . . 36 


\section{List of Tables}

2.1 Case matrix for the sub-model sensitivity study $\ldots \ldots$. . . 18

2.2 Case matrix for the Urea-SCR dosing chamber . . . . . . . 22

2.3 Sensitivity to number of parcels . . . . . . . . . . . 22 



\title{
List of Abbreviations
}

\author{
DEF Diesel Exhaust Fluid \\ RANS Reynolds Averaged Navier Stokes \\ CCGEx Competence Center of Gas Exchange \\ SCR Selective Catalyst Reduction \\ NOx Mono nitrogen oxides \\ PDPA Phase Doppler Particle Analyzer \\ LDV Laser Doppler Velocimetry \\ LPT Lagrangian Particle Tracking \\ DRW Discrete Random Walk
}





\section{List of Symbols}

\begin{tabular}{lll}
$\rho$ & density & $\mathrm{kg} / \mathrm{m}^{3}$ \\
$\rho_{p}$ & particle density & $\mathrm{kg} / \mathrm{m}^{3}$ \\
$U_{i}$ & mean velocity component & $\mathrm{m} / \mathrm{s}$ \\
$u_{i}$ & instantaneous velocity component & $\mathrm{m} / \mathrm{s}$ \\
$u_{p, i}$ & particle instantaneous velocity component & $\mathrm{m} / \mathrm{s}$ \\
$K$ & turbulent kinetic energy & $\mathrm{m}^{2} / \mathrm{s}^{2}$ \\
$\nu$ & kinematic viscocity & $\mathrm{m}^{2} / \mathrm{s}$ \\
$\nu_{T}$ & turbulent viscocity & $\mathrm{m}^{2} / \mathrm{s}$ \\
$u_{\tau}$ & friction velocity & $\mathrm{m} / \mathrm{s}$ \\
$F_{D}$ & drag force per unit mass & $\mathrm{N} / \mathrm{kg}$ \\
$T_{\infty}$ & continous phase local temperature & $\mathrm{K}$ \\
$T_{p}$ & particle temperature & $\mathrm{K}$ \\
$T_{v a p}$ & particle vaporization temperature & $\mathrm{K}$ \\
$T_{b p}$ & particle boiling temperature & $\mathrm{K}$ \\
$m_{p}$ & mass of particle & $\mathrm{kg}$ \\
$c_{p}$ & heat capacity & $\mathrm{J} / \mathrm{kg}-\mathrm{K}$ \\
$A_{p}$ & surface area of the particle & $\mathrm{m})$ \\
$h$ & convective heat transfer coefficient & $\mathrm{W} / \mathrm{m}^{2}-\mathrm{K}$ \\
$\epsilon_{p}$ & particle emissivity & 1 \\
$\sigma_{b}$ & Stefan-Boltzmann constant $\left(=5.67 \cdot 10^{-8}\right)$ & $\mathrm{W} / \mathrm{m}^{2}-\mathrm{K}^{4}$ \\
$\theta_{R}$ & radiation temperature $\left(\frac{G}{4 \sigma}\right)$ & $\mathrm{K} / 4$ \\
$\widehat{T}$ & integral time scale & $\mathrm{K}$ \\
$\widehat{T_{L}}$ & lagrangian integral time scale & $\mathrm{s}$ \\
$l$ & characteristic length scale & $\mathrm{s}$ \\
$\sigma$ & surface tension & $\mathrm{m}$ \\
$U_{r e l}$ & relative velocity & $\mathrm{N} / \mathrm{m}$ \\
$D_{p}$ & diameter of particle & $\mathrm{m} / \mathrm{s}$ \\
$\mu_{g}$ & gas dynamic viscosity & $\mathrm{m}$ \\
$U_{g}$ & gas velocity & $\mathrm{kg} /(\mathrm{ms})$ \\
$a$ & distance & $\mathrm{m} / \mathrm{s})$ \\
$P$ & power & $\mathrm{m}$ \\
$\omega$ & angular frequency & $\mathrm{W}\left(\mathrm{J} \mathrm{s} \mathrm{s}^{-1}\right)$ \\
& & $\mathrm{rad}$ \\
& \\
\hline &
\end{tabular}





\section{Chapter 1}

\section{Introduction}

The demand on the automotive and logistics industry for finding new more environmentally friendly technology has increased significantly over the recent years. The competitive advantage of being eco-friendly has motivated companies to invest more money on research for finding solutions on how to leave a smaller ecological footprint. For combustion engines the use of catalysts has traditionally been a popular way to decrease hazardous substances.

The Selective Catalyst Reduction (SCR) is the process of neutralizing nitrogen oxides (NOx) with the aid of a catalyst substance. It was first applied in thermal power plants in Japan in the late 1970s but was adopted as late as the mid 2000s for mobile diesel engines. Today the technology has been adopted to both commercial diesel truck applications as well as light-duty vehicles like cars.

With continuously new standards being formulated decreasing the NOx limits even further the development of SCR technology is essential for manufacturers to retain competitive advantages.

\subsection{Technical background}

SCR diesel technology uses a catalyst system to break down dangerous NOx emissions produced by diesel engines into nitrogen and water. The chemical reactions used in SCR systems require a constant feed of ammonia gas. In automotive applications SCR delivers ammonia using a mixture between urea and deionized water also called Diesel Exhaust Fluid (DEF). DEF is sprayed into the exhaust stream by an advanced injection system and when vaporizing it decomposes to form ammonia and carbon dioxide. Thereafter the ammonia reacts with the NOx and forms nitrogen and water.

The amount of DEF needed for decreasing the NOx and meet the limiting standards is dependant on several characteristics of the engine such as exhaust mass flow rate and temperature. Diesel engines manufacturers continuously work on developing their SCR technology to become more efficient.

\subsection{Motivation and structure}

Academical papers studying the applicability of CFD models for Urea-SCR catalysts are limited. The reason may be that complete process behind the 
Urea-SCR technology involves many different physical phenomena which in return makes it difficult to form proper validation assessments of modelling techniques. As a result this paper will not look into the numerical reactions but instead focus on the evaporation and mixing of the spray.

The aim of this paper is to first conduct a systematic study of the available modelling framework for evaporating sprays in order to identify its advantages and limitations in simulations of the urea SCR injection. Secondly the knowledge inherited will be applied on a real Urea-SCR case where evaporation and mixing effects are studied. This is done by studying spatial averages of droplets size and velocity distributions which also makes results easily comparable with experimental results. Due to the lack of studies on the spray inside an Urea-SCR dosing chamber another application in terms of a Water mist nozzle (used in Water Mist Firefighting Systems) will be investigated for the study of the numerical framework.

Figure 1.1 concludes the working process starting with the sensitivity study of sub-models using the Water mist nozzle case. The knowledge about the sub-models is thereafter applied to the Urea-SCR case together with boundary conditions supplied by Scania.

The two cases are similar in several ways:

- Both applications involve evaporating sprays with similar droplet sizes

- Small breakup length, $D_{\text {droplet }} \ll D_{\text {oriface }}$

- Similar Weber number $W e_{\text {mist }} \sim W e_{S C R} \sim 1$

- Similar Stokes number $S t K_{m i s t} \sim S t K_{S C R} \sim 1$

- Similar Ohnsorge number $O h_{m i s t} \sim O h_{S C R} \ll 1$

Urea-SCR

Water mist

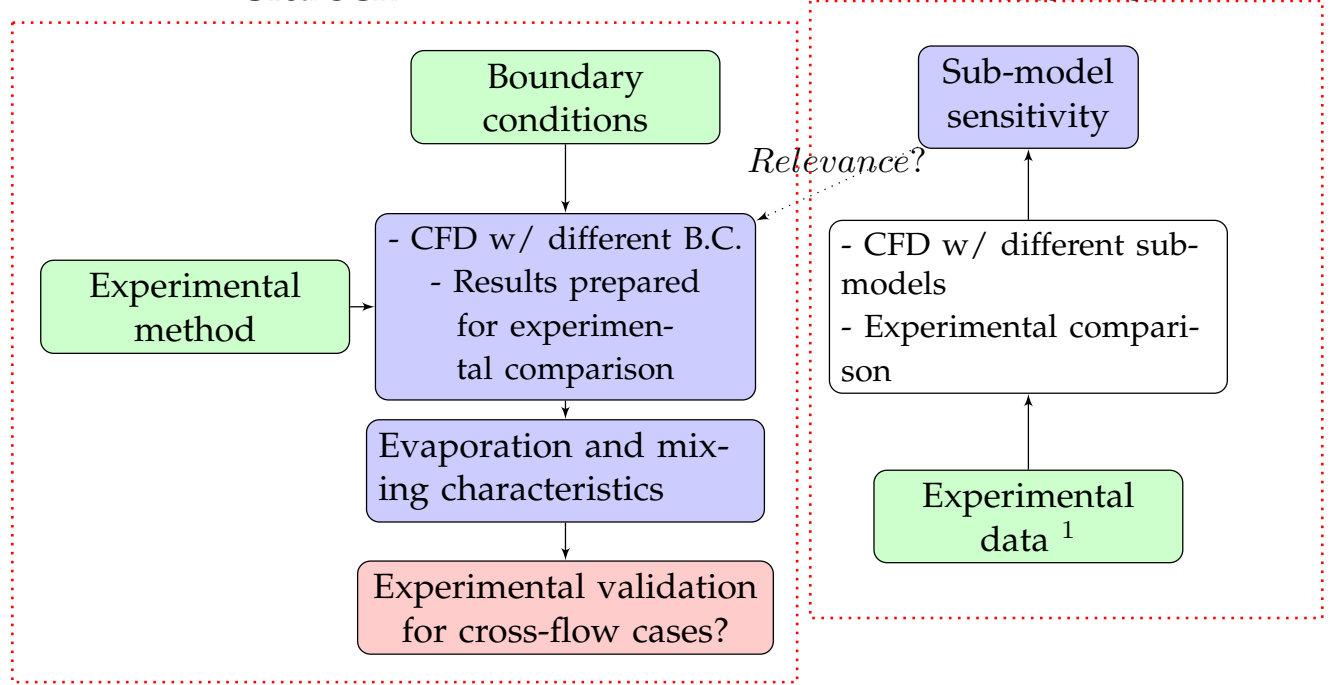

FIGURE 1.1: Work flow, ${ }^{1}$ Iannantuoni et al., 2013 


\subsection{Literature review}

Iannantuoni et al., 2013 conducted both numerical and experimental work on a water mist nozzle. They used experimental techniques in terms of Laser Doppler Velocemitry (LDV) and Phase doppler anemometry (PDA) to capture droplet size and velocity distributions at certain downstream distances from the nozzle. The experimental results and methodology from their study are used as a basis for the model sensitivity study.

For the Urea-SCR application Nova and Tronconi, 2014 have completed a full overview on the modelling framework. Their book covers both the technology as a commercial system (markets, legislations, standards etc) but also from a more fundamental perspective on the actual technological processes. Nova and Tronconi, 2014 has involved several technical experts from both industry and academia and the book provides a first example of a framework for modelling the gas flow process in SCR. Even though CFD techniques only covers a small part of the book it provides its readers with the basics around multi-phase CFD techniques such as Lagrangian Particle Tracking which includes evaporation phenomena. A more general approach to the CFD framework have been documented by Prosperetti and Tryggvarson, 2007 which is used throughout this work as general CFD handbook. Furthermore as this work is conducted with a commercial CFD software the theory guide written by the software developers (see Fluent Theory Guid, 2013) is constantly used for finding model references.

On the experimental side of things Tropea et al Tropea et al., 2007 provides a comprehensive handbook for experimental techniques applicable in this project. 



\section{Chapter 2}

\section{Models and methods}

This Method chapter describes the theory behind the numerical framework and thereafter the actual numerical setup for the different cases as well as a methodology behind which cases are to be investigated.

\subsection{Numerical procedure}

This chapter gives a brief overview of the models used for the numerical simulations.

\subsubsection{Governing equations}

The governing equations for incompressible fluid flow are the Navier Stokes equations, i.e. conservation of mass and momentum:

$$
\begin{gathered}
\frac{\partial \rho}{\partial t}+\frac{\partial}{\partial x_{j}}\left[\rho u_{j}\right]=0 \\
\frac{\partial\left(\rho u_{i}\right)}{\partial t}+\frac{\partial\left[\rho u_{i} u_{j}\right]}{\partial x_{j}}=-\frac{\partial p}{\partial x_{i}}+\frac{\partial \tau_{i j}}{\partial x_{j}}+\rho f_{i}+S_{i}
\end{gathered}
$$

where $\tau_{i j}$ is deviatoric stress tensor. In addition when heat transfer is included conservation of energy equation is needed to close the system of equations.

$$
\rho \frac{\partial e}{\partial t}+\rho \frac{\partial u_{j} e}{\partial x_{j}}=-\frac{\partial Q_{i}}{\partial x_{i}}-p \frac{\partial u_{i}}{\partial x_{i}}+\frac{\partial \tau_{i j} u_{i}}{\partial x_{j}}
$$

where $e$ is the internal (thermal) energy due to microscopic motion and $Q_{i}$ heat flux usually approximated by Fick's law of heat conduction $Q_{i}=$ $-K \frac{\partial T}{\partial x_{i}}$ where $K$ is the heat conductivity.

\subsubsection{Finite volume method}

The Finite volume method (FVM) was chosen as discretization method of the partial differential governing equations. The method refers to a domain filled with "finite volumes" which are spatially fixed. By using the divergence theorem volume integrals are converted to surface integrals which may be calculated algebraically as fluxes on each "finite volume". The Finite volume method has for long be seen as favourable within the field of Computational Fluid Dynamics (CFD) as the method is conservative and can easily be adopted on unstructured meshes. 


\subsubsection{Turbulence model}

The conventional way of dealing with turbulence is through averaging the flow quantities over time. All quantities are reformulated by Reynolds decomposition where each scalar or vector is subdivided into an averaged part $\bar{\Phi}_{i}$ and a fluctuating part $\Phi_{i}^{\prime}$ :

$$
\Phi_{i}=\bar{\Phi}_{i}+\Phi_{i}^{\prime}
$$

by applying Reynolds Decomposition to the incompressible Navier Stokes equations one obtains:

$$
\frac{\partial U_{i}}{\partial t}+U_{j} \frac{\partial U_{i}}{\partial x_{j}}=-\frac{1}{\rho} \frac{\partial P}{\partial x_{i}} \frac{\partial}{\partial x_{j}}\left(\nu \frac{\partial U_{i}}{\partial x_{j}}-\overline{u_{i}^{\prime} u_{j}^{\prime}}\right)
$$

where the Reynolds stress tensor is identified as $\rho \overline{u_{i}^{\prime} u_{j}^{\prime}}$. The Boussinesq hypothesis assumes that the turbulent stress can be expressed by single point mean flow quantities. By applying

$$
-\rho \overline{u_{i}^{\prime} u_{j}^{\prime}}=\rho \nu_{T}\left(\frac{\partial U_{i}}{\partial x_{j}}+\frac{\partial U_{j}}{\partial x_{i}}\right)-\frac{2}{3} \rho K \delta_{i j}
$$

where $\nu_{T}$ is a property of the flow named the turbulent viscosity. The value of $\nu_{T}$ is modelled by an order of magnitude assumption :

$$
\nu_{T} \sim \Lambda V
$$

where $\Lambda$ is a length scale and $V$ a velocity scale of the large eddies. In order to model these scales one need quantities which are independent on the reference frame. The turbulent kinetic energy $K$ and the turbulent dissipation rate $\epsilon$ are chosen. The dissipation rate is a quantity describing the small scale transformation into heat. However since the energy cascade from the mean flow into heat heat is assumed to be in equilibrium $\epsilon$ may be used for describing the length of the large eddies. By dimensional analysis $\nu_{T}$ may be found as:

$$
\nu_{T}=C_{\mu} \frac{K^{2}}{\epsilon}
$$

where $C_{\mu}$ is a function of mean strain and rotation rates, the angular velocity of the system rotation, and the turbulence fields Shih et al., 1995. Since turbulent kinetic energy $K$ and turbulent dissipation $\epsilon$ are introduced two additional transport equations for these quantities are needed. The transport equation for $K$ consists of four terms:

$$
\frac{D K}{d t}=P_{K}+P_{K, b}-\epsilon-\frac{\partial T_{j, K}}{\partial x_{j}}
$$

where the right hand side represents production from mean flow $P_{K}$, buoyancy production $P_{K, b}$, dissipation rate $\epsilon$ and spatial redistribution of kinetic energy $T_{j, k}$. The exact formulation becomes:

$$
\frac{D K}{d t}=2 \nu_{T} S_{i j} S_{j i}+G_{b}-\epsilon+\frac{\partial}{\partial x_{j}}\left(\left(\nu+\frac{\nu_{T}}{\sigma_{K}}\right) \frac{\partial K}{\partial x_{j}}\right)
$$


where $G_{b}$ is the generation of turbulence kinetic energy due to buoyancy and $\sigma_{k}=1.0$. The terms in the transport equation for the dissipation of energy can in a similar way be formulated as:

$$
\frac{D \epsilon}{d t}=P_{\epsilon}+P_{\epsilon, b}-D-\frac{\partial T_{j, \epsilon}}{\partial x_{j}}
$$

Where $P_{\epsilon}$ is production, $P_{\epsilon_{b}}$ buoyancy production, $D$ destruction, and $T_{j, \epsilon}$ spatial redistribution of $\epsilon$. The final transport equation for $\epsilon$ is formulated as:

$$
\frac{D \epsilon}{d t}=C_{1} S_{\epsilon}+C_{1 \epsilon} \frac{\epsilon}{K} C_{3 \epsilon} G_{b}-C_{2} \frac{\epsilon^{2}}{K+\sqrt{v \epsilon}}+\frac{\partial}{\partial x_{j}}\left(\left(\nu+\frac{\nu_{T}}{\sigma_{\epsilon}}\right) \frac{\partial \epsilon}{\partial x_{j}}\right)
$$

where the standard values for the coefficients are $C_{1 \epsilon}=1.44, C_{2}=$ const, and $\sigma_{\epsilon}=1.3 . C_{1}$ is a function of the turbulence fields and the strain rate tensor. The major difference in comparison with Standard $K-\epsilon$ is how production $\left(P_{\epsilon}\right)$ and destruction $(D)$ are modelled. The production term $\left(P_{\epsilon}\right)$ does not involve the turbulent kinetic energy but uses the formulation of $C_{1}$ together with the strain rate tensor. The destruction term $(D)$ does not have the singularity for $K \rightarrow 0$ which could cause numerical stability issues for the Standard $K-\epsilon$ model. This turbulence model is named the Realizable $K-\epsilon$ model by Shih et al., 1995 .

\subsubsection{Boundary layer}

Viscosity and no slip boundary conditions will cause momentum diffusion due to shear stresses. The region affected are along the walls of the internal flow and up to a certain wall normal distance. This region is called boundary layer. Considering incompressible flows only the boundary layer properties may be characterised depending on the flow regime, i.e. laminar or turbulent. For the turbulent boundary layer a non-dimensionalized flow profile may be derived along a flat plate as a function of the two nondimensionalized variables:

$$
\begin{aligned}
y^{+} & =\frac{u_{\tau} y}{\nu} \\
U^{+} & =\frac{U}{u_{\tau}}
\end{aligned}
$$

where $u_{\tau}$ is the friction velocity. Without looking into the derivation the final result gives two significant limits depending on the wall normal distance. These are the viscous sublayer and the logarithmic layer. The sublayer is valid for $1<y^{+}<5$. The loglayer is applicable for $30<y^{+}$. The transition between these regions is called the buffer layer, $5<y^{+}<30$, where no distinguishable mathematical law applies. 


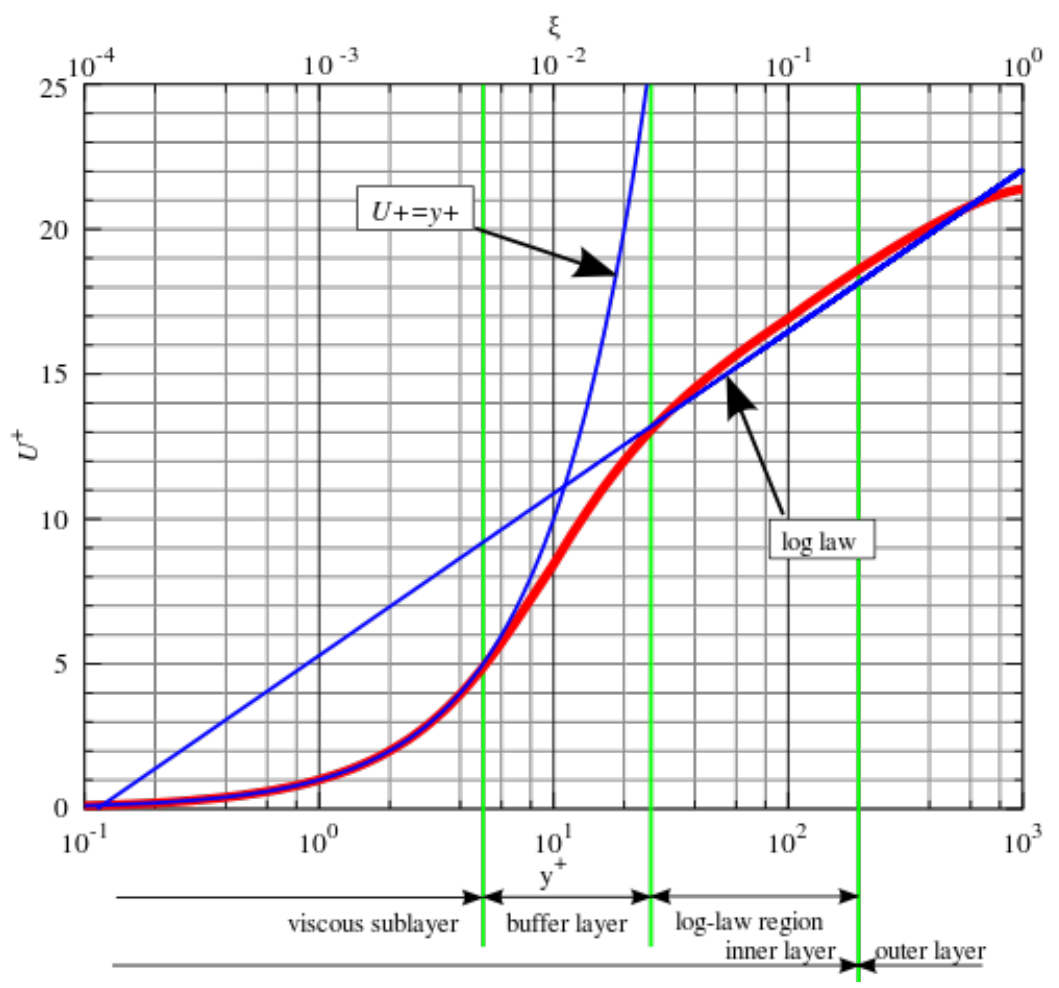

FIGURE 2.1: The turbulent boundary layer

\section{Near wall modelling}

The modelling of the boundary layer may need special care depending on the choice of turbulence modelling. As noted in equation $2.12 \mathrm{~K}$ is located in the denominator, in the region close to the wall $K \rightarrow 0$ which will result in $\frac{D \epsilon}{d t} \rightarrow \infty$. This means that for the k-epsilon turbulence model one needs to adopt additional models depending on the $y^{+}$. A blending function (Kader, 1981) between low Reynolds number model and wall functions is employed for the modelling the near-wall flow. The method is referred to as Enhanced Wall Treatment in Fluent and is considered flexible to mesh properties as it can model a larger span of $y^{+}$.

\subsubsection{Lagrangian Particle Tracking}

\section{The Eulerian/Lagrangian description}

Lagrangian Particle Tracking for multiphase flows follows the Euler/Lagrange approach where the continous phase is treated as a continuum following the Navier-Stokes equations and the discrete phase is solved by tracking a large number of droplets through the continuous flow field. The dispersed phase can exchange momentum, mass, and energy with the fluid phase.

\section{Basic principle}

The continous phase is solved by the Navier-Stokes equation and RANS Realizable k-epsilon model. Depending on the choice of coupling as mentioned in section 2.1.5 the discrete phase may have a source term in the mass, momentum and energy equation for the eulerian phase. The discrete phase is solved numerically by various sub-models depending on the flow. 
While the particle motion equation is the most obvious additional models for breakup, evaporation, turbulent dispersion and coalescence of particles can be applied.

\section{Particle motion and interaction with continuous phase}

The trajectory of an individual particle is predicted by the force balance equation of the particle:

$$
\frac{d u_{i, p}}{d t}=F_{D}\left(u_{i}-u_{i, p}\right)+\frac{g_{i}\left(\rho_{p}-\rho\right)}{\rho_{p}}+F_{i}
$$

Where $F_{D}\left(u_{i}-u_{i, p}\right)$ is the particle drag force per unit particle mass and:

$$
F_{D}=\frac{18 \mu}{\rho_{p} d_{p}^{2}} \frac{C_{D} R e_{r}}{24}
$$

where $R e_{r}$ is the relative Reynolds number:

$$
R e_{r} \equiv \frac{\rho d_{p}\left|u_{i, p}-u_{i}\right|}{\mu}
$$

Note that depending on the inclusion of Turbulence or not the instantaneous velocity $u_{i}$ may replaced by the mean velocity $U_{i}$. See section 2.1.3 and 2.1.5. The last term $F_{i}$ incorporates virtual mass and pressure gradient forces which becomes important when $\frac{\rho}{\rho_{p}} \rightarrow 1$. Throughout this study $\frac{\rho}{\rho_{p}} \ll 1$ and these forces can be seen as negligible.

\section{Parcel representation of particles}

Lagrangian Particle Tracking techniques in CFD requires the computer to store physical quantities for each individual particle or droplet. When the total number of particles or droplets reach high orders it is therefore of interest to reduce the computational effort by introducing so called particle parcels. Each particle parcel contains a statistical representation of a certain number of individual particles. This method is especially valuable when it comes to collision calculations as presented by O'Rourke, 1981 but is also valuable when simply calculating particle trajectories.

\section{Sub models}

In addition to the particle trajectory equations several sub models can be applied to describe additional physical phenomena affecting the particles.

\subsection{Drag}

The spherical drag law proposed by Morsi and Alexander, 1972 is formulated for smooth particles:

$$
C_{D}=a_{1}+\frac{a_{2}}{R e}+\frac{a_{3}}{R e^{2}}
$$

Where $a_{1}, a_{2}$ and $a_{3}$ are constants varying over certain ranges of $R e$. 


\subsection{Heat transfer and evaporation}

The heat transfer and vaporization laws applied are dependant on the particle temperature $T_{p}$, vaporization temperature $T_{v a p}$ and the boiling point temperature $T_{b p}$ of the liquid .

\section{Passive range}

In the passive range $T_{p}<T_{\text {vap }}$ the temperature of the particle is modelled through a heat balance including the convective heat transfer and the absorption/emission of radiative heat transfer.

$$
m_{p} c_{p} \frac{d T_{p}}{d t}=h A_{p}\left(T_{\infty}-T_{p}\right)+\epsilon_{p} A_{p} \sigma_{b}\left(\theta_{r}^{4}-T_{p}^{4}\right)
$$

where:

$$
\begin{aligned}
& m_{p}=\text { mass of particle }(\mathrm{kg}) \\
& c_{p}=\text { heat capacity }(\mathrm{J} / \mathrm{kg}-\mathrm{K}) \\
& A_{p}=\text { surface area of the particle }\left(\mathrm{m}^{2}\right) \\
& T_{\infty}=\text { local temperature of the continuous phase }(\mathrm{K}) \\
& h \quad=\text { convective heat transfer coefficient }\left(\mathrm{W} / \mathrm{m}^{2}-\mathrm{K}\right) \\
& \epsilon_{p}=\text { particle emissivity }(1) \\
& \sigma_{b}=\text { Stefan-Boltzmann constant }\left(5.67 \cdot 10^{-8} \mathrm{~W} / \mathrm{m}^{2}-\mathrm{K}^{4}\right) \\
& \theta_{R}=\text { radiation temperature }\left(\frac{G}{4 \sigma}\right)^{1 / 4}(\mathrm{~K})
\end{aligned}
$$

\section{Vaporization range}

For the range $T_{v a p} \leq T_{p}<T_{b p}$ vaporization is activated. Since the evaporation rate is assumed to be high the effect of convection for evaporated material outside the droplet becomes important (Stefan flow). The following expression for the mass transfer which takes into account convective effects was proposed by Miller et al., 1998 and Sazhin, 2006:

$$
\frac{d_{m p}}{d t}=k_{c} A_{p} \rho_{\infty} \ln \left(1+B_{m}\right)
$$

where $k_{c}$ is the mass transfer coefficient given by the Sherwood number correlation by Ranz and Marshall, 1952:

$$
S h_{A B}=\frac{k_{c} d_{p}}{D_{i, m}}=2.0+0.6 R e_{d}^{1 / 2} S c^{1 / 3}
$$

where $B_{m}$ is the Spalding mass number formulated as:

$$
B_{m}=\frac{Y_{i, s}-Y_{i, \infty}}{1-Y_{i, s}}
$$

where $Y_{i, s}$ and $Y_{i, \infty}$ are the vapor mass fractions respectively for the droplet surface and the bulk gas.

\section{Boiling range}

In the range $T_{p} \geq T_{b p}$ the boiling rate equation by Kuo, 1986 is formulated as:

$$
\frac{d\left(d_{p}\right)}{d t}=\frac{4 k_{\infty}}{\rho_{p} c_{p, \infty} d_{p}}\left(1+0.23 \sqrt{R e_{d}}\right) \ln \left[1+\frac{c_{p, \infty}\left(T_{\infty}-T_{p}\right)}{h_{f g}}\right]
$$


where:

$$
\begin{aligned}
& c_{p, \infty}=\text { heat capacity of the gas }(\mathrm{J} / \mathrm{kg}-\mathrm{K}) \\
& \rho_{p}=\text { droplet density }\left(\mathrm{kg} / \mathrm{m}^{3}\right) \\
& k_{\infty}=\text { thermal conductivity of the gas }(\mathrm{W} / \mathrm{m}-\mathrm{K})
\end{aligned}
$$

\subsection{Turbulence}

In Reynolds Averaged Navier Stokes (RANS) models the mean flow field is solved while the fluctuating part of the instantaneous velocity is modelled through the Boussinesq hypothesis (see equation 2.6). Since the instantaneous velocity field is unknown the particle trajectories are calculated based on the mean flow field, see section 2.1.5. In order to account for the influence of turbulence on the lagrangian phase the concept of a stochastic model (random walk model) is applied.

\section{The integral time scale}

The concept of an integral time scale $\widehat{T}$ for the turbulent eddies is introduced:

$$
\widehat{T}=\int_{0}^{\infty} \frac{u_{p}^{\prime}(t) u_{p}^{\prime}(t-\tau)}{\overline{u_{p}^{\prime 2}}} d \tau
$$

it can be shown that the integral time scale is proportional to the particle dispersion rate such that the particle diffusivity is given as $\overline{u_{i}^{\prime} u_{j}^{\prime}} \widehat{T}$. For tracer particles (small mass and no drift) the integral time becomes the Lagrangian integral time $\widehat{T_{L}}$ which can be approximated as:

$$
\widehat{T_{L}}=C_{L} \frac{k}{\epsilon}
$$

where $C_{L}$ is an unknown constant. The Lagrangian integral time $\widehat{T_{L}}$ is thereafter matched with the scalar diffusion rate predicted by the model $\nu_{t} / \sigma$. Note that this is the same scalar diffusion rate as in the k-equation, see equation 2.10. For the k-epsilon model one will obtain:

$$
T_{L}=0.15 \frac{k}{\epsilon}
$$

\section{Discrete random walk model}

The interaction between a particle and a turbulent eddie of the eulerian phase is modeled by the Discrete Random Walk (DRW) model as formulated by Gosman and Ioannides, 1983. The model assumes that a turbulent eddie can be characterized by gaussian distributed velocity fluctuation and a time scale which is proportional to the integral time scale, see equation 2.26 .

\subsection{Breakup}

Three different breakup models were applied for modelling the secondary breakup of droplets: the Wave model, the Kelvin Helmholtz Rayleigh Taylor (KHRT) model and the Stochastic Secondary Breakup (SSD) model. The dimensionless number which governs the secondary breakup is the Weber number formulated as: 


$$
W e=\frac{\rho_{p} U_{r e l}^{2} l}{\sigma}=\frac{\text { inertia }}{\text { surface tension }}
$$

where:

$$
\begin{aligned}
\rho_{p} & =\text { density of particle }\left(\mathrm{kg} / \mathrm{m}^{3}\right) \\
U & =\text { the relative velocity }(\mathrm{m} / \mathrm{s}) \\
l & =\text { characteristic length scale, typically diameter }(\mathrm{m}) \\
\sigma & =\text { surface tension }(\mathrm{N} / \mathrm{m})
\end{aligned}
$$

\section{Wave}

The work by Reitz, 1987 proposes that breakup time and the new droplet size are related to the fastest-growing Kelvin-Helmholtz instability. This model is applicable to $W e>100$ where the Kelvin-Helmholtz instability is believed to dominate the breakup. The fastest growing instability is derived by a jet stability analysis as described by Reitz and Bracco, 1986.

\section{KHRT}

The KHRT is based on the work by Beale and Reitz, 1999 and Patterson and Reitz, 1998. It combines the effects by Kelvin-Helmholtz instabilities and Rayleigh- Taylor instabilities due to aerodynamic acceleration. By tracking wave growth on the droplet surface from both instability mechanisms the break up will occur where the fastest growing wave is located based on local conditions. For droplets travelling close to the nozzle region a Levich core length is applied which limits the KHRT model to only take into account Kelvin-Helmholtz instabilities, Levich, 1962.

\section{SSD}

The Stochastic Secondary Droplet (SSD) model was first introduced by Apte et al., 2003. The model assumes breakup as a discrete random event returning in a new distribution of diameter scales. In the SSD model the secondary droplet size is determined by solving the Fokker-Planck equation for the probability density function. Model parameters for the statistical models are adjusted based on the local conditions.

\section{TAB}

The Taylor Analogy Breakup (TAB) model is based on Taylor's analogy Taylor, 1963 where an oscillating and distorting droplet is represented as a spring mass system.

\subsection{Collisions and Coalescence}

Possible collisions between droplets scale with $\sim N^{2}$ where $N$ is the total number of droplets. Since all possible collisions need to be calculated for each time step one needs to reduce the number of possible collisions in order to minimize computational effort. O'Rourke, 1981 proposed an algorithm which efficiently reduces the computational cost of a spray simulation. First the method suggests that possible collisions are scaled by a stochastic measurement. Secondly collisions can only appear between parcels if they are located in the same continuous phase cell. If two parcels are determined to have a collision the method further calculates the type of collision based on the collisional Weber number formulated as: 


$$
W e_{c}=\frac{\rho_{p} U_{r e l}^{2} \bar{D}}{\sigma}
$$

where $\bar{D}$ is the arithmetic mean diameter of the two parcels. The outcome of the collision can either be coalescence or bouncing it is calculated from the angle of the collision as well as the collisional Weber number. More specifically the parameters are variables for the calculation of a collision parameter which depending on a critical value will determine the outcome.

2.1.5.0.6 Wall film model The Lagrangian droplet boundary conditions for the walls inside the dosing chamber require special modelling. Throughout this work the model used for droplets colliding with walls is that they form thin films. There are several physical phenomena which all may be applied on the formation of the film which include evaporation, splashing, heat-transfer, shear forces and film breakup as displayed in figure 2.2. For further explanation on the modelling techniques refer to Fluent Theory Guide, 2013.

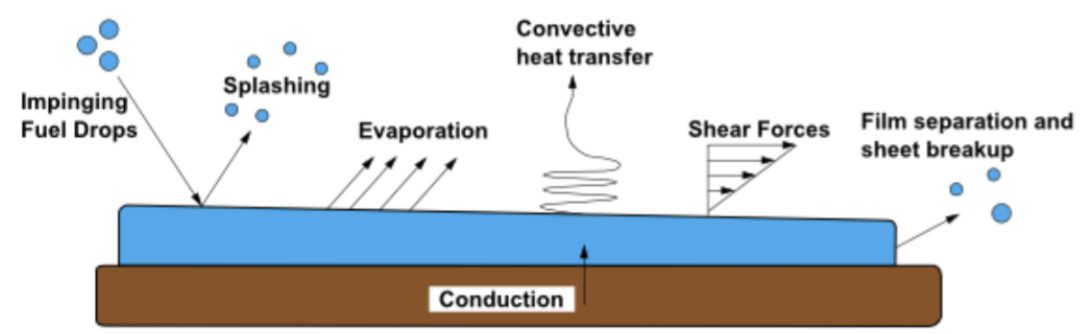

FIGURE 2.2: Physical phenomena around the liquid film

\section{Solution procedure}

The ANSYS Fluent package (v14.5) together with IcemCFD mesh modeler was chosen as software for this work. They are both commercial software commonly used in industry.

The solution for the discrete phase means integration in time of the force balance equation 2.15. During this time models for heat and mass transfer are calculated together with breakup laws and collisions as described in section 2.1.5. The interaction between the discrete phase and the continuous phase can be either one or two-way coupled which means that quantities such as mass, momentum and energy can be transferred one or two-way. A dimensionless number characterizing the behaviour (in terms of trajectory i.e. momentum) of discrete particles in a fluid flow is the Stokes number defined as the ratio of the characteristic time of a particle (or droplet) to a characteristic time of the flow or of an obstacle:

$$
S t K=\frac{\rho_{p} D_{d}^{2} U}{18 \mu_{g} D}
$$

where: 


$$
\begin{aligned}
\rho_{p} & =\text { density of particle }\left(\mathrm{kg} / \mathrm{m}^{3}\right) \\
D_{p} & =\text { diameter of particle }(\mathrm{m}) \\
U & =\text { the gas velocity }(\mathrm{m} / \mathrm{s}) \\
\mu_{g} & =\text { gas dynamic viscosity }(\mathrm{kg} /(\mathrm{m} \mathrm{s})) \\
D & =\text { characteristic dimension of the obstacle }(\mathrm{m})
\end{aligned}
$$

If $S t K>>1$ particles will detach from the flow, if $S t K<<1$ particles follow fluid flow streamlines closely. Depending on the application a two way coupling may be needed for all physical quantities but for the cases in this study the exchange of momentum between the phases is the most critical one. By developing the concept of using the Stokes number as an indicator for momentum coupling sensitivity CFD handbooks have come up with a more explanatory momentum coupling parameter:

$$
\prod_{m o m}=\frac{C}{1+S t K}=\frac{\dot{m}_{p} / \dot{m}_{g}}{1+S t K}
$$

where $C$ is the mass flow rate fraction. The parameter indicates that for small stokes number the mass flow rate fraction is the driving variable for the parameter. For $\prod_{m o m} \ll 1$ a two way coupling is not needed.

\subsection{One or two-way coupling solution procedure}

The solution procedure for one and two-way coupling are presented in figure 2.3 and figure 2.4. For simulations that involve particle breakup the discrete phase must be tracked with the fluid flow time step, this means essentially that $\Delta t$ remains constant and equal for the two phases.

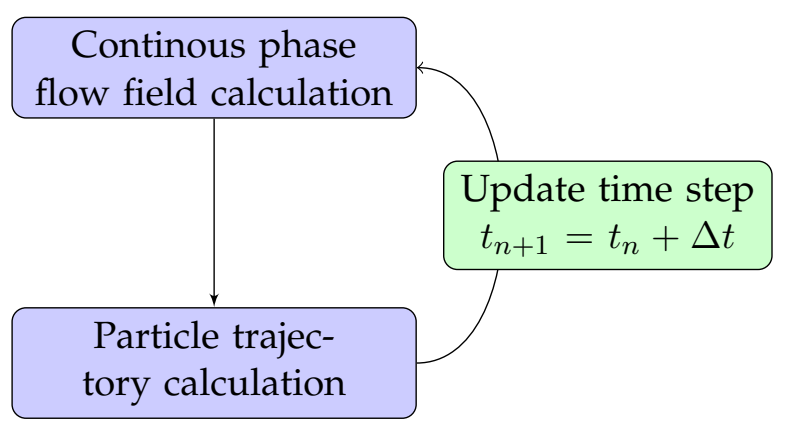

FIGURE 2.3: Solution procedure one-way coupled calculations 


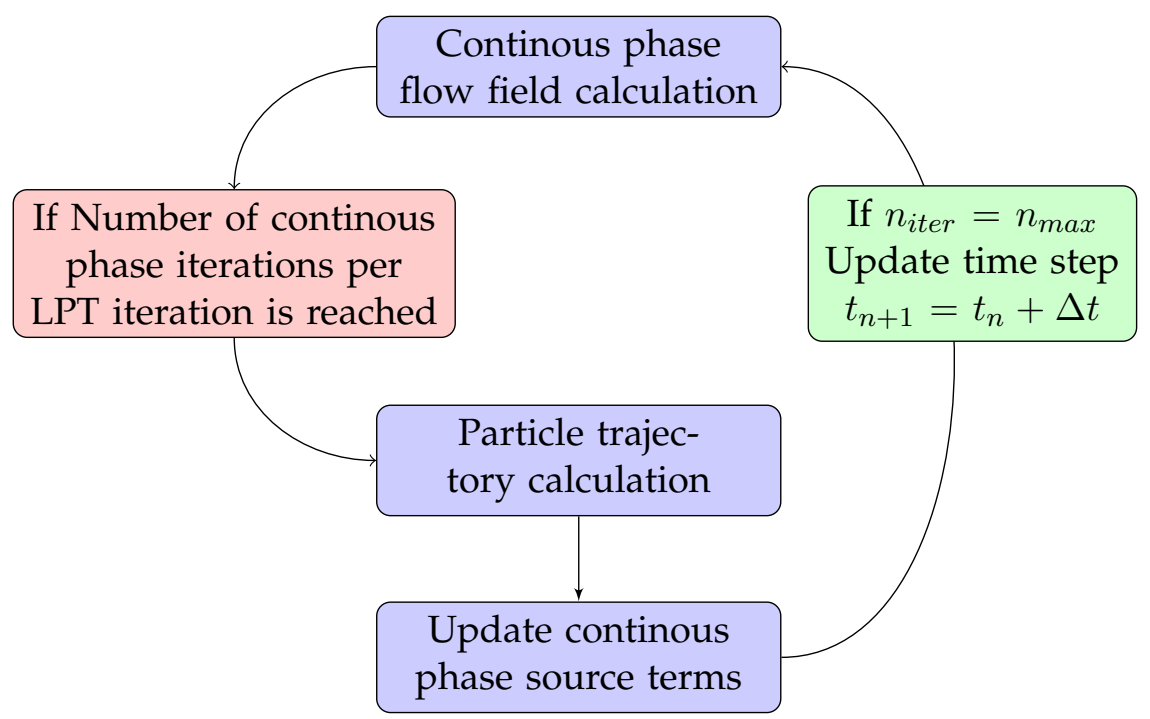

FIGURE 2.4: Solution procedure two-way coupled calculations

\subsection{Experimental procedure}

On the experimental side of things Tropea et al Tropea et al., 2007 provides a comprehensive handbook for experimental techniques applicable in this project. A well established technique which is based upon a coherent laser light is Phase Doppler Anemometry (PDA), see figure 2.5. In addition to Laser Doppler Velocimetry (LDV) which only captures the frecuency shift PDA also captures the phase shift Ferrer, 2010. Since the phase shift is related to the droplet diameter PDA has the ability to measure both velocity and droplet size data under some hypotheses.

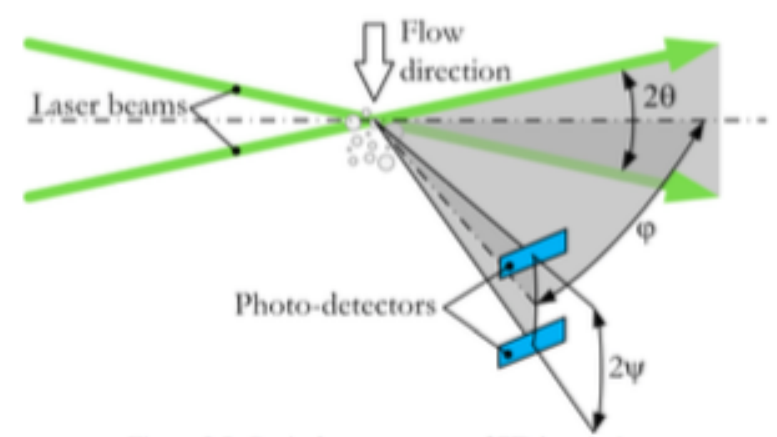

FIGURE 2.5: Optical arrangement of PDA technique 


\subsection{Case setup}

This chapter explains the numerical setup used for the two different cases.

\subsubsection{Pre study - Water mist nozzle}

The motivation for the pre study is to assess the numerical models for drag, coalescence, turbulence, breakup and evaporation. Since experimental results as well as injection properties are available the case can provide some insights on the sensitivity of sub-models for the numerical results.

\section{Computational domain}

The computational domain of the water mist spray is cone shaped (see figure 2.6) with upper diameter $D_{1}=2 m=3600 D_{\text {nozzle }}$ and lower diameter $D_{2}=4 m=7200 D_{\text {nozzle }}$. Total height $H_{\text {mist }}=2 \mathrm{~m}=3600 D_{\text {nozzle. }}$. The gas phase is stationary so the "walls" of the domain are representing external boundaries with constant pressure where flow in both directions may occur. The top wall is seen as a wall with a no slip velocity condition. By approximating the width of the spray from the injection cone angle the geometry is created in such way that the domain diameter is always four times larger than the actual spray in order to minimize the effect of the boundary conditions on the actual spray flow. The gas temperature is assumed to be $25^{\circ} \mathrm{C}$ as the experimental results by the previous study was taken in normal room temperatures.

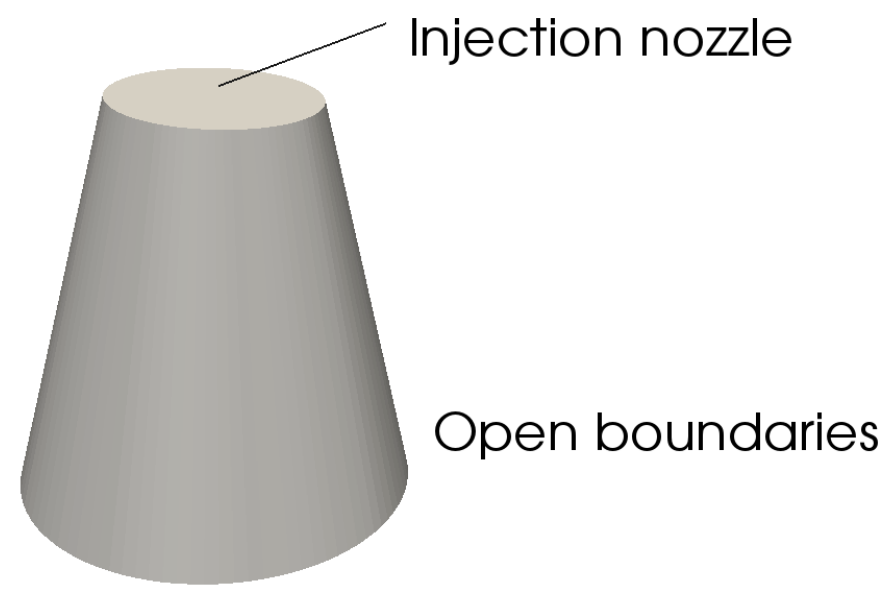

FIGURE 2.6: The dosing chamber unit

\section{Discretization}

The mesh structure is an O-grid with a first cell height (close to the injector) of $10^{-} 3 \mathrm{~m} \approx 20 D_{\text {droplet }}$ which a growth rate of 1.05 per vertical layer downstream. The mesh is displayed in figure 2.7. 


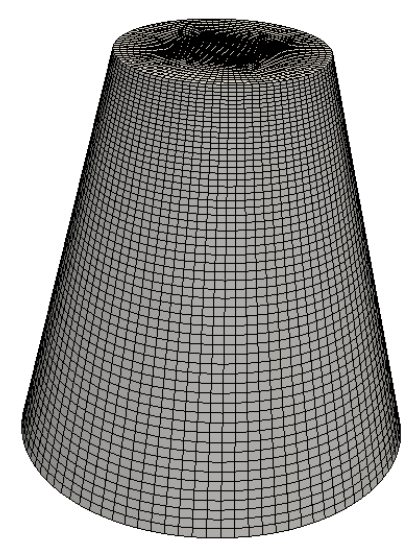

FIGURE 2.7: O-grid mesh

\section{LPT properties}

The injection properties are defined in accordance with the work of Iannantuoni et al., 2013:

- Mean diameter $\mu=60 \mu m$

- Weibull distribution with shape parameter $n=2.1$

- Initial velocity $140 \mathrm{~m} / \mathrm{s}$

- Cone half angle $=25 \mathrm{deg}$

- Mass flow rate $=0.033 \mathrm{~kg} / \mathrm{s}(21 / \mathrm{min})$

- 250000 particle parcels / second

A Stokes number of $S t K \approx 1.5$ indicates that the spray will influence the gas phase and a two-way coupling is needed. Due to the initially stationary gas phase it is not possible to formulate the momentum coupling parameter. However with a two way coupling and open boundaries (w/ constant pressure) a gas flow will develop due to the spray movement.

Parcel description: for every time step a constant number of parcels with equal mass are injected (based on mass flow rate and number of parcels/s). Each parcel represents a number of droplets with equal diameter where the diameter is drawn from the Weibull distribution. As a result parcels with different diameters will initially represent a different number of droplets. Each parcel is injected with a velocity of $140 \mathrm{~m} / \mathrm{s}$ at an vertical angle between 0-25 degrees (drawn from uniform distribution) and an axial angle between 0-360 degrees. This will represent a full cone injection.

Different combinations of sub-models for the droplets are executed in order to assess the applicability of different sub-models for drag, turbulence, coalescence, breakup and evaporation. 


\section{Solution setup}

The eulerian phase is solved transiently with a PISO (Pressure Implicit with Splitting of Operator) algorithm, Issa, 1986. Time step of $\Delta t=5 \cdot 10^{-4}$ gives a Courant number of $C o \sim 1$ and time integration is conducted with an implicit scheme. Pressure, momentum, energy and species are solved using second order upwind interpolation schemes. The calculation runs for 100000 time steps which gives a simulation time of $1.0 \mathrm{~s}$. Preliminary calculations has showed statistical convergence for the spray velocity and diameters at already $0.5 \mathrm{~s}$.

\section{Case matrix}

Three different sub-model categories were chosen to be analysed:

- DPM-Turbulence coupling (Discrete random walk model, none)

- Evaporation (convective, diffusive)

- Droplet breakup (SSD, Wave, KHRT, TAB)

The cases were narrowed down to seven different ones in order to distinguish effects for each category alone as per 2.1.

\begin{tabular}{|c|c|c|c|c|c|c|c|c|}
\hline \multirow{2}{*}{ Case } & \multicolumn{2}{|c|}{ DPM-Turbulence } & \multicolumn{3}{|c|}{ Evaporation } & \multicolumn{4}{|c|}{ Droplet breakup } \\
\hline & Yes & No & Diffusive & Convective/diffusive & SSD & Wave & KHRT & TAB \\
\hline 1 & $\mathrm{x}$ & & $\mathrm{x}$ & & & & & \\
\hline 2 & & $\mathrm{x}$ & $\mathrm{x}$ & & & & & \\
\hline 3 & $\mathrm{x}$ & & $\mathrm{x}$ & & $\mathrm{x}$ & & & \\
\hline 4 & $\mathrm{x}$ & & & $\mathrm{x}$ & $\mathrm{x}$ & & & \\
\hline 5 & $\mathrm{x}$ & & & $\mathrm{x}$ & & $\mathrm{x}$ & & \\
\hline 6 & $\mathrm{x}$ & & & $\mathrm{x}$ & & & $\mathrm{x}$ & \\
\hline 7 & $\mathrm{x}$ & & & $\mathrm{x}$ & & & & $\mathrm{x}$ \\
\hline
\end{tabular}

TABLE 2.1: Case matrix for the sub-model sensitivity study 


\subsubsection{Urea-SCR dosing chamber}

\section{Computational domain}

The geometrical domain consists of an inlet and outlet fixed to a pipe respectively, see figure 2.8. In the beginning of the center box section the injector is placed. Since the box is slightly larger than the standard pipe diameter a diverging (close to inlet) and (close to outlet) section of the chamber is needed.

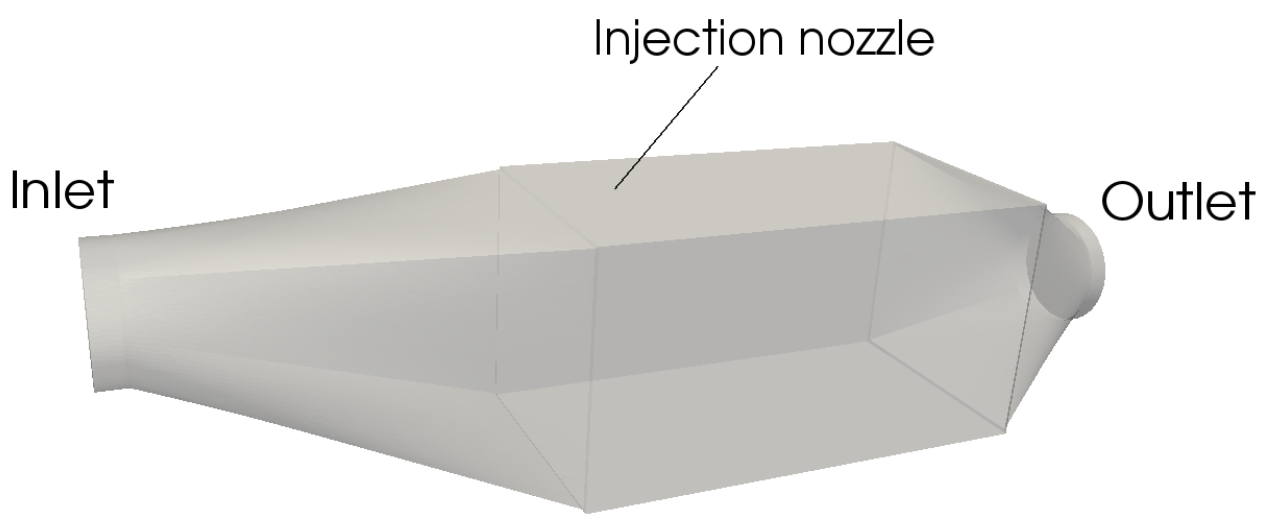

FIGURE 2.8: The dosing chamber unit

The geometry has been supplied by Scania and is designed in such way that experimental tests can be conducted with it as one of the walls can be replaced by a flat glass. The box shape is solely due to the need for having a flat glass as the experimental techniques are based on refractions of light. Real conventional dosing chamber does not have this box shape and look more like a pipe.

\section{Discretization}

In difference from the pre study case the Urea-SCR dosing chamber case is an internal flow where the spray operates in a cross stream gas flow. Since the gas flow will be subject to walls boundary layers will develop. As mentioned in section 2.1.4 a blending function between the low Reynolds number model and wall functions are applied which depends on the wall normal distance to the first cell $\left(y^{+}\right)$. The near-wall flow is not assumed to affect the spray significantly so it is therefore preferred to use the blending function since resolving the near-wall flow would increase computational cost. Using a first layer thickness of $10^{-4} \mathrm{~m}$ and five prism layers with a 1.2 growth rate the non-dimensional wall normal distance $y^{+} \sim 1$. 

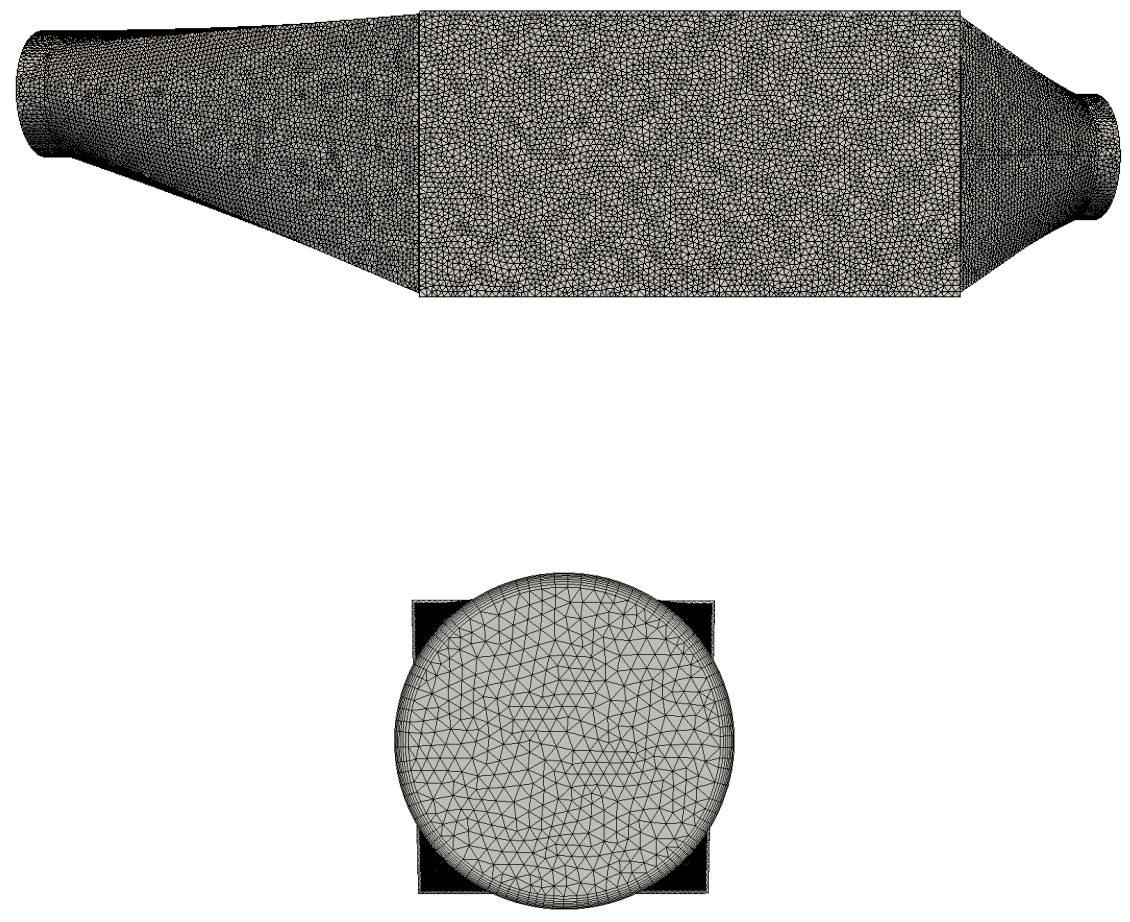

FIGURE 2.9: Mesh of dosing chamber, unstructured mesh with 5 prism layers

\section{Boundary conditions}

The boundary conditions for the gas (Eulerian) phase were determined assuming a developed pipe flow at the inlet. CFD simulations of periodic (steady) pipe flow with an equal geometry and mass flow rate provided velocity, $\mathrm{K}$ and $\epsilon$ profiles which were imported as inlet conditions for the dosing chamber. Since cases involve different running temperatures each inlet condition needed to be calculated uniquely. This is despite assuming an isothermal solution due to varying air (exhaust gas) properties in terms of viscosity and density. The outlet was defined as a pressure outlet allowing reversed flow and the walls with a no slip boundary condition.

The Lagrangian phase is set up using a wall-film model for collisions with walls. When a particle parcel hits the outlet it will vanish. The Eulerian (gas) phase is assumed to be air and the Lagrangian phase water.

\section{LPT properties}

Injection properties are defined based on the data supplied by Scania. Additional experiments for validating the injection properties are to be performed. Size distribution data provided by Scania are presented in fig 3.9. 


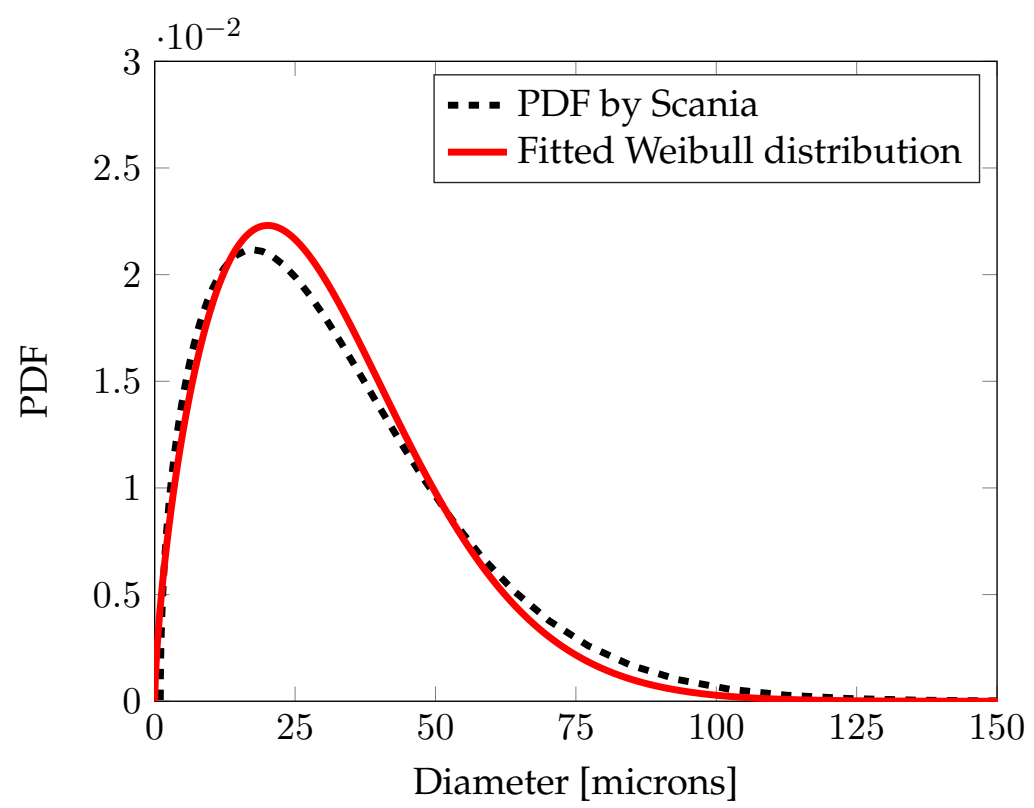

FIGURE 2.10: Fitted Weibull distribution to experimental data

- Mean diameter $\mu=34 \mu m$

- Weibull distribution with shape parameter $n=1.6$

- Initial velocity $25 \mathrm{~m} / \mathrm{s}$

- Cone half-angle $=50$ degrees

- Mass flow rate $=195 \mathrm{~g} / \mathrm{min}$

- 200000 particle parcels/second

With a Stokes number of $S t K \approx 0.4$ two way coupling can't be neglected. But due to a small relative mass flow rate the momentum coupling parameter $\prod_{m o m} \approx 0.02$ it may be assumed that the spray will have a very small impact on the overall gas flow. A one-way coupling is chosen but but simulations with two-way coupling will also be conducted for investigating sensitivity.

\section{Solution setup}

The setup is nearly identical to the water mist case, see section 2.3.1. Slightly different time step $\Delta t=10^{-4}$. 5000 time steps which gives a simulation time of $0.5 \mathrm{~s}$. By studying droplets residence times (mild cross-flow) the simulation time will give at least around 20 droplet flow-throughs thus stable conditions are assumed.

\section{Case matrix}

No cross flow simulations in ambient conditions (case 1a) were conducted in order to validate the size distribution data provided by Scania and the injector manufacturer. Since the numerical simulation of the spray is heavily dependant on the injection properties it is of great importance to make sure 
the input data is in accordance with reality. Additional cases which correspond to normal running conditions are presented in table 2.2. Note that mild and high cross-flow nomenclature refers to the different mass flow rates as per table 2.2.

\begin{tabular}{|l|l|l|l|}
\hline & Case & Mass flow [kg/h] & Temperature [C] \\
\hline No cross flow & 1a & 0 & 20 \\
\hline \multirow{3}{*}{ Mild cross flow } & 2a & 400 & 200 \\
\cline { 2 - 4 } & 2b & 400 & 300 \\
\cline { 2 - 4 } & 2c & 400 & 400 \\
\hline \multirow{3}{*}{ High cross flow } & $4 a$ & 1400 & 200 \\
\cline { 2 - 4 } & $4 \mathrm{~b}$ & 1400 & 300 \\
\cline { 2 - 4 } & 4c & 1400 & 400 \\
\hline
\end{tabular}

TABLE 2.2: Case matrix for the Urea-SCR dosing chamber

Additionally an assessment of the parcel sensitivity is to be performed corresponding to table 2.3.

\begin{tabular}{|l|l|}
\hline Case & Parcels / s \\
\hline $4 \mathrm{~b}$ & 200000 \\
\hline 4b_s & 1000000 \\
\hline
\end{tabular}

TABLE 2.3: Sensitivity to number of parcels 


\section{Chapter 3}

\section{Results and discussion}

The result section is divided into the model sensitivity study (of water mist nozzle) and the Urea-SCR dosing chamber.

\subsection{Sensitivity to sub-models - Water mist nozzle case}

Numerical simulations were performed for investigating the sensitivity of sub-models for the Lagrangian Particle Tracking model. Different sub-models were used for secondary break-up, evaporation and turbulence. Results from CFD were compared with experimental results by Iannantuoni et al., 2013. The injection properties of the spray were defined equivalently to the injection properties by Iannantuoni et al., 2013 as per section 2.3.1. The methodology used in their study for displaying results is also applied by means of sampling droplets on a plane located at a downstream distance of $h=1 m=1800 D_{\text {nozzle }}$ (see figure 3.1). The number averaged size and velocity are thereafter calculated as a function of the radial distance from the centerline (assuming axisymmetry).

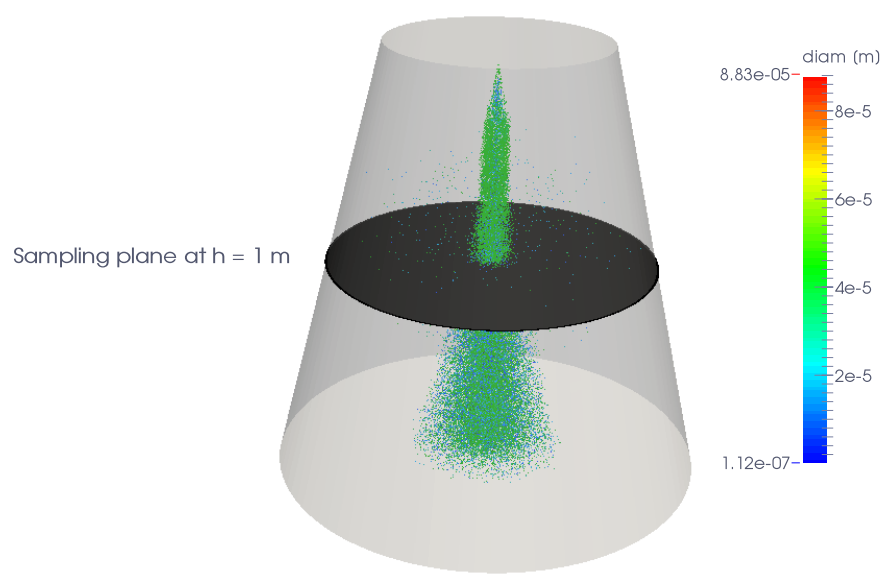

FIGURE 3.1: Droplets coloured by diameter at $\mathrm{t}=0.8 \mathrm{~s}$ after injection start

Each case in table 2.1 was computed and the effects of sub-models for the categories of evaporation, turbulence coupling and droplet breakup are presented below. The plots $x$-axis shows the radial distance from the center normed by nozzle diameter. The y-axis shows the number averaged droplet diameter (normed by nozzle diameter) and velocity (normed by injection 
velocity). The black dashed plot shows experimental results by Iannantuoni et al., 2013.

\subsubsection{Turbulence}

The turbulence coupling used for calculating droplet trajectories as per section 2.1.5 affects the results significantly. Without turbulence coupling the spatial droplet size distribution doesn't reach a similar state as the experimental data as noted in figure 3.2. Neglect the order of magnitude as no breakup model was activated. The droplet velocity distribution is also affected by the turbulence coupling (see figure 3.3) as the case run with the coupling corresponds better to the experimental data.

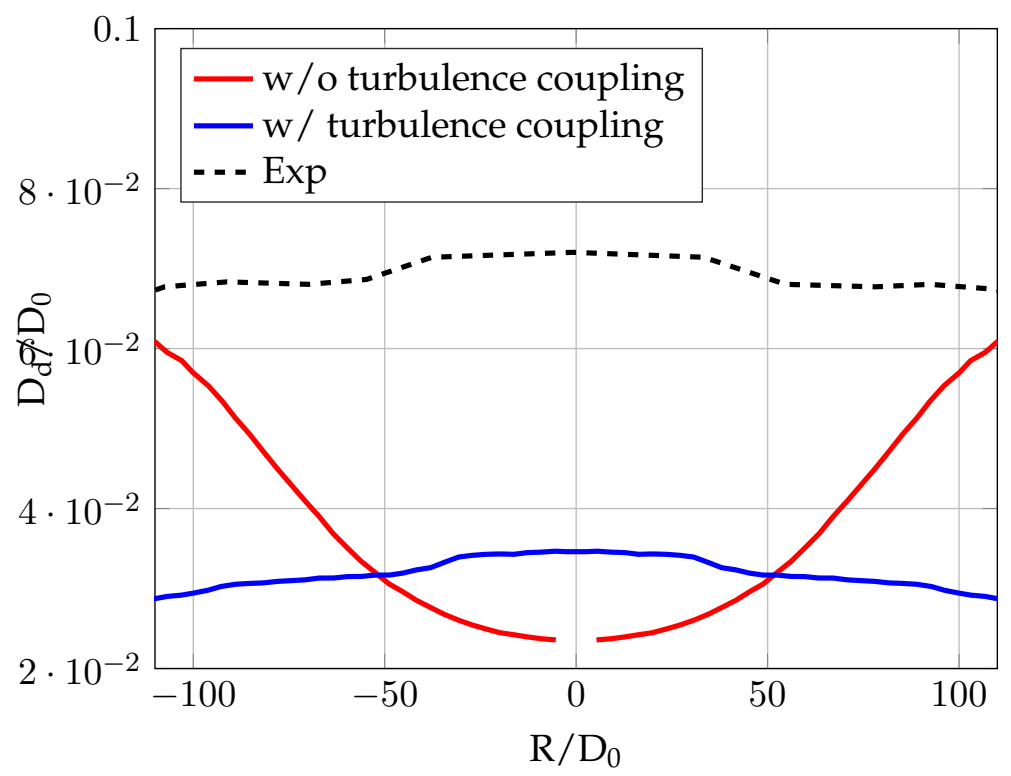

FIGURE 3.2: Number averaged radial size distribution for Discrete Random Walk turbulence coupling vs none (case 1 \& 2) 


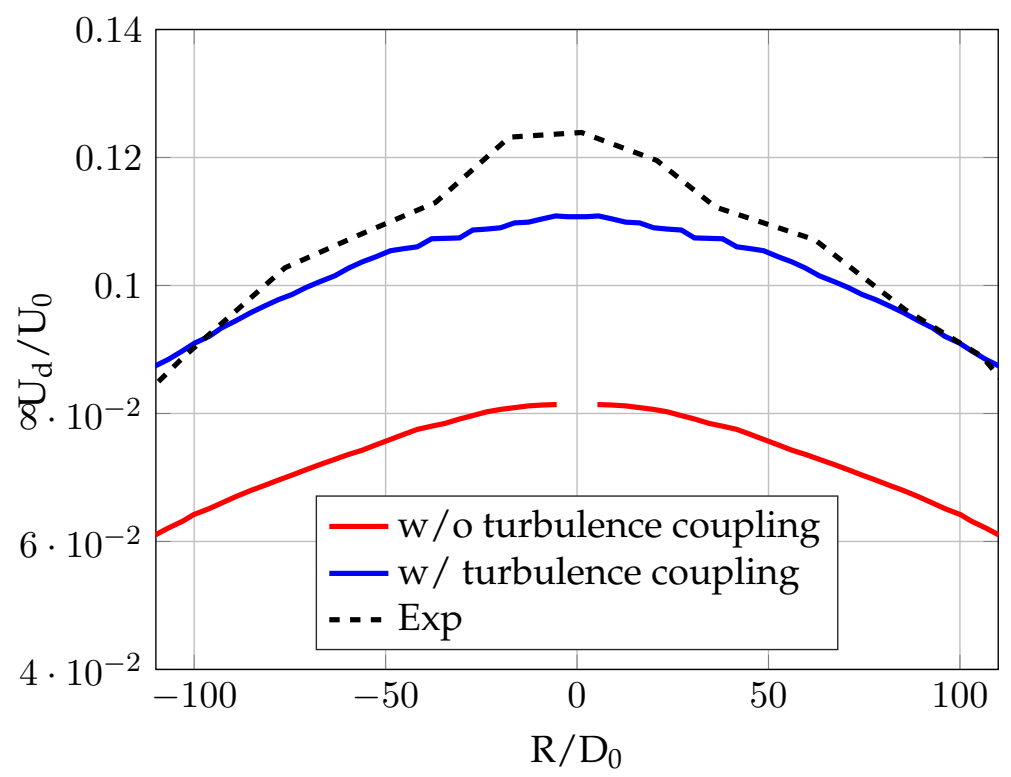

FIGURE 3.3: Number averaged radial velocity distribution for Discrete Random Walk turbulence coupling vs none (case $1 \& 2$ )

\subsubsection{Evaporation}

As the water mist case is conducted at normal room temperature $\left(25^{\circ} \mathrm{C}\right)$ evaporation rates are low and Stefan flow effects Miller et al., 1998 can be neglected which in return means that the convective effects are not dominating the evaporation. As seen in figure 3.4 and 3.5 it is not possible to distinguish any significant differences as the evaporation can be seen as a pure diffusion process. The diffusive/convective model becomes the pure diffusive model as the evaporation rates reach smaller values.

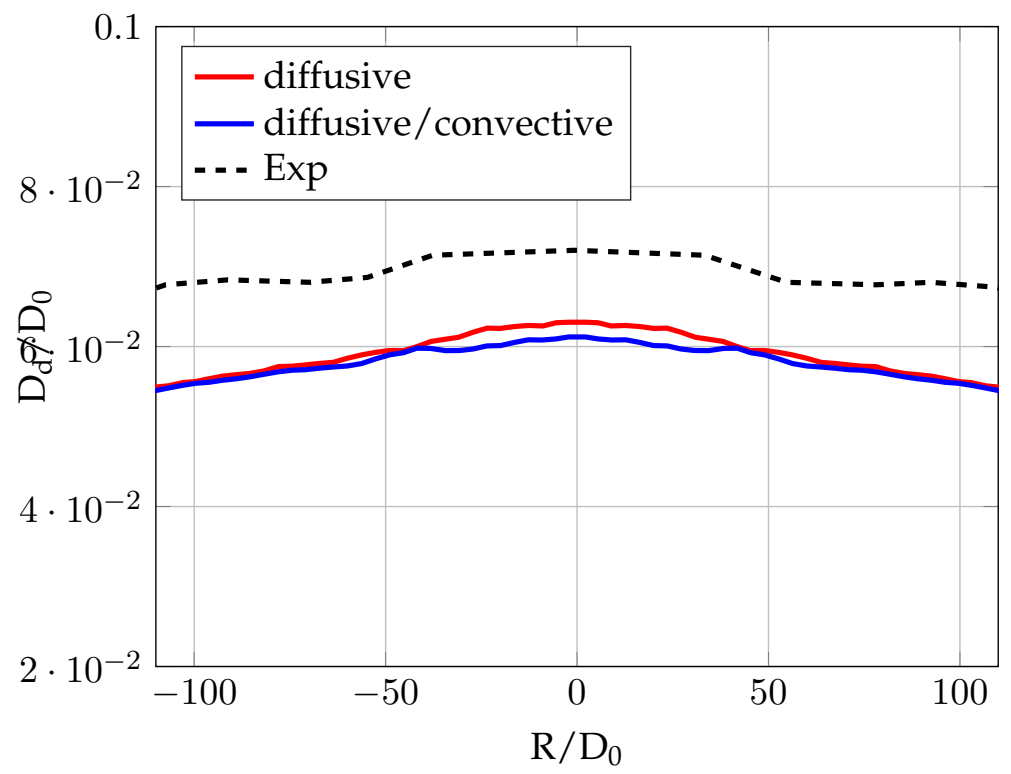

FIGURE 3.4: Number averaged radial size distribution for different evaporation models (case $3 \& 4$ ) 


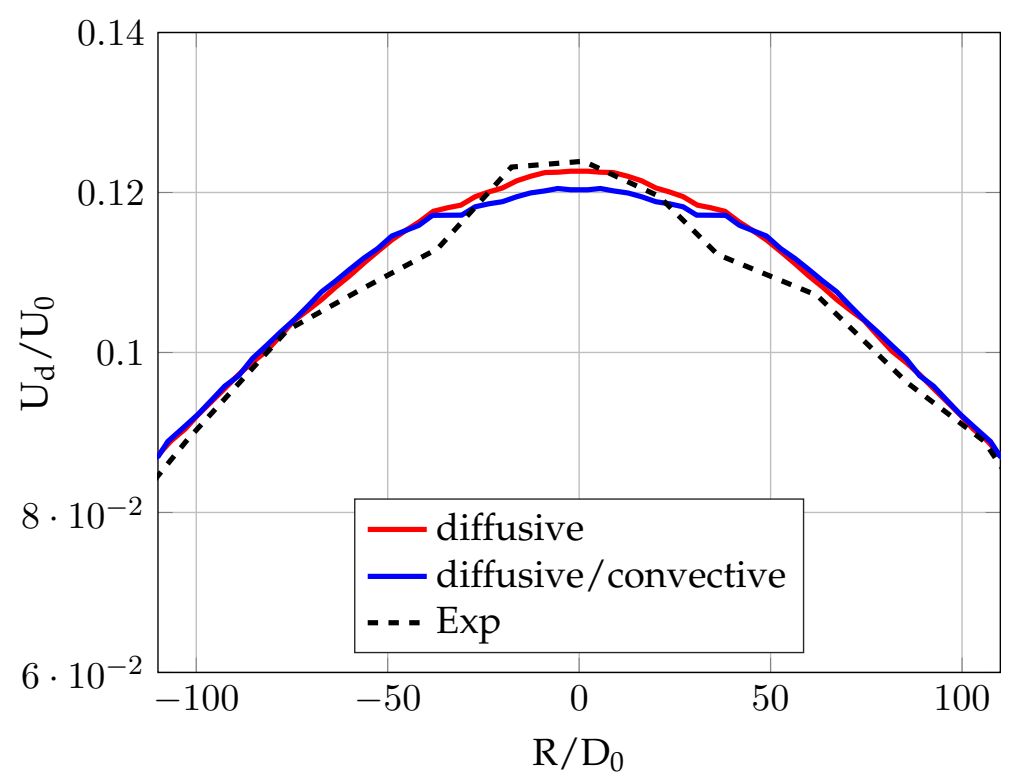

FIGURE 3.5: Number averaged radial velocity distribution for different evaporation models (case $3 \& 4$ )

\subsubsection{Breakup}

The effect of different breakup models is displayed in figure 3.6 and 3.7. It is noticed that KHRT and Wave give similar results. As the KHRT model basically is a hybrid version of the Wave model (see section 2.1.5) this is expected. It is also expected that the TAB model underestimates the droplet size distribution since the model is known for overestimating the shattering of droplets when the Weber number is in the higher limit $(W e \sim 10)$ of the models applicability range Taylor, 1963.

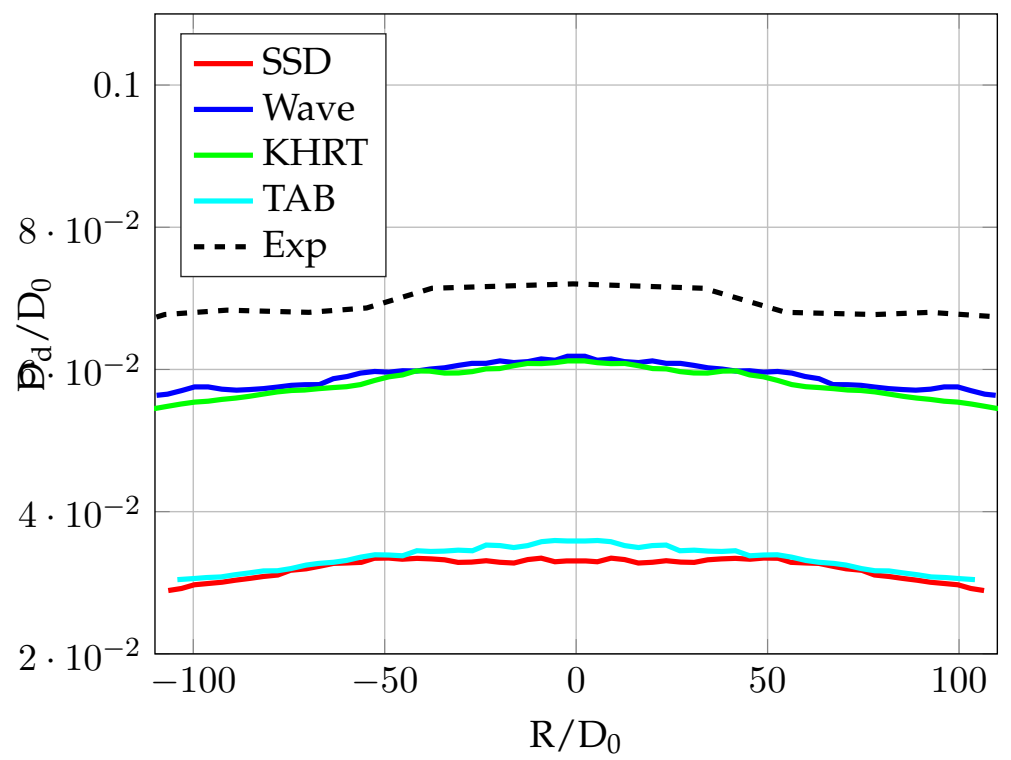

FIGURE 3.6: Number averaged radial size distribution for different breakup models (case 5, 6, 7 \& 8) 


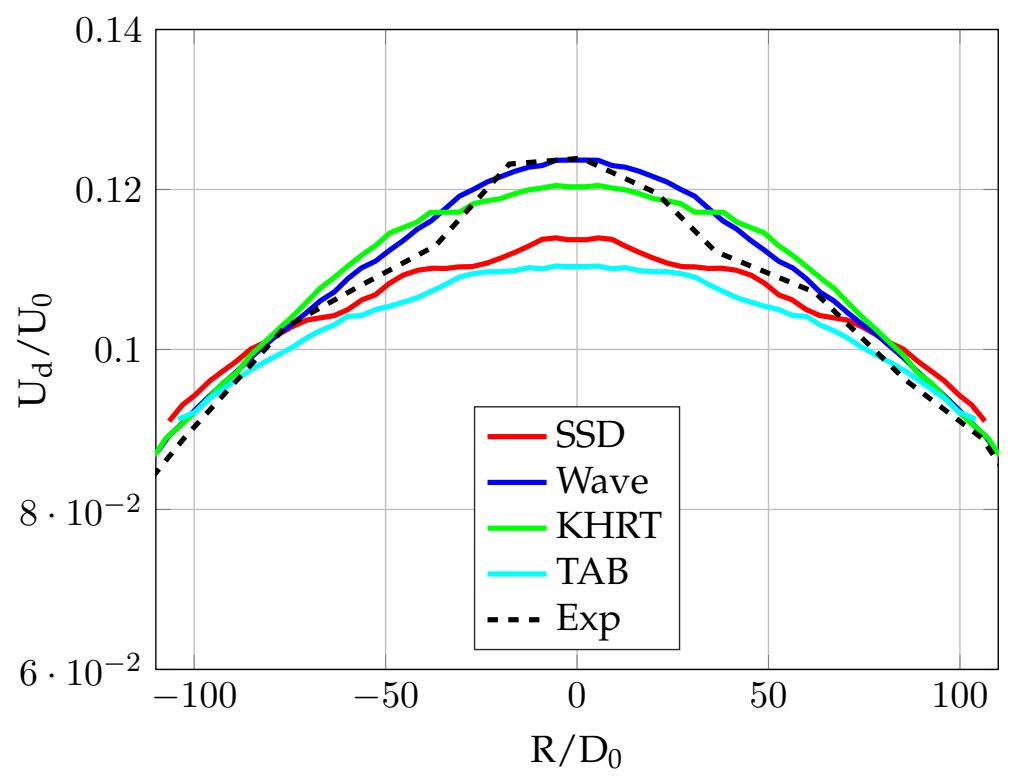

FIGURE 3.7: Number averaged radial velocity distribution for different breakup models (case 5, 6, 7 \& 8)

\subsubsection{Summary of sensitivity study}

The sensitivity analysis of the water mist nozzle results in the following conclusions:

- Turbulence coupling essential for the calculation of droplet trajectories

- Stefan Flow effect may be neglected (see Miller et al., 1998). However the additional computational cost for also taking convective effects into account is small so the diffusive/convective model is favourable

- The Wave breakup model corresponds to the experimental results best (size distribution more sensitive than velocity)

The case which shows the best correspondence to the experimental results seems to be case 5 in table 2.1. The Urea-SCR simulations are therefore set up with similar conditions. 


\subsection{Urea-SCR dosing chamber}

The results for the Urea-SCR dosing chamber are divided into injection properties validation (no cross-flow case), mass flow rate effects (also referred to as cross-flow effects, see section 2.3.2, temperature effects and parcel sensitivity. Droplet size and velocity number averaged distributions are sampled along three vertical center lines at 1D, 2D and 3D distance downstream as per figure 3.8 where $D$ is taken as the inlet diameter of $D=12 \mathrm{~cm}$. The height of the chamber is denoted $H$.

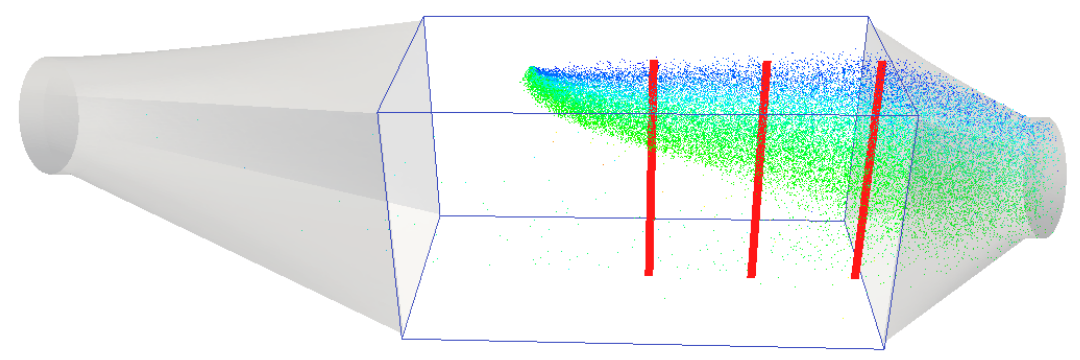

FIGURE 3.8: Sampling lines at $\mathrm{L}=1 \mathrm{D}, 2 \mathrm{D}$ and 3D downstream of the injector where $\mathrm{D}$ is the inlet diamater $D=$ $12 \mathrm{~cm}$

\subsubsection{Validation of size distribution}

For the no cross-flow case (zero velocity at $T=25 C^{\circ}$ ) only experimental results (conducted by Giovanni Lacagnina in combustion engines lab) are compared with the injection properties supplied by the nozzle manufacturer.

Droplet sizes were sampled experimentally along a $20 \mathrm{~cm}$ horizontal line at different downstream distances from the injector. The normalized PDF from the experimental measurements are compared with the data measured by Scania in figure 3.9. A quite coarse correspondence is noted for the dataset measured close to the nozzle $(2 \mathrm{~cm}$ and $4 \mathrm{~cm})$. However further downstream the measurement $(18 \mathrm{~cm})$ seems to correspond better to the PDF provided by Scania. These results increases the uncertainties regarding the spray calculations since the injection properties for the numerical work were taken from the PDF by Scania (see Fitted Weibull distribution). Ideally the size distribution as close as possible to the nozzle should be used as injection properties for numerical simulations. 


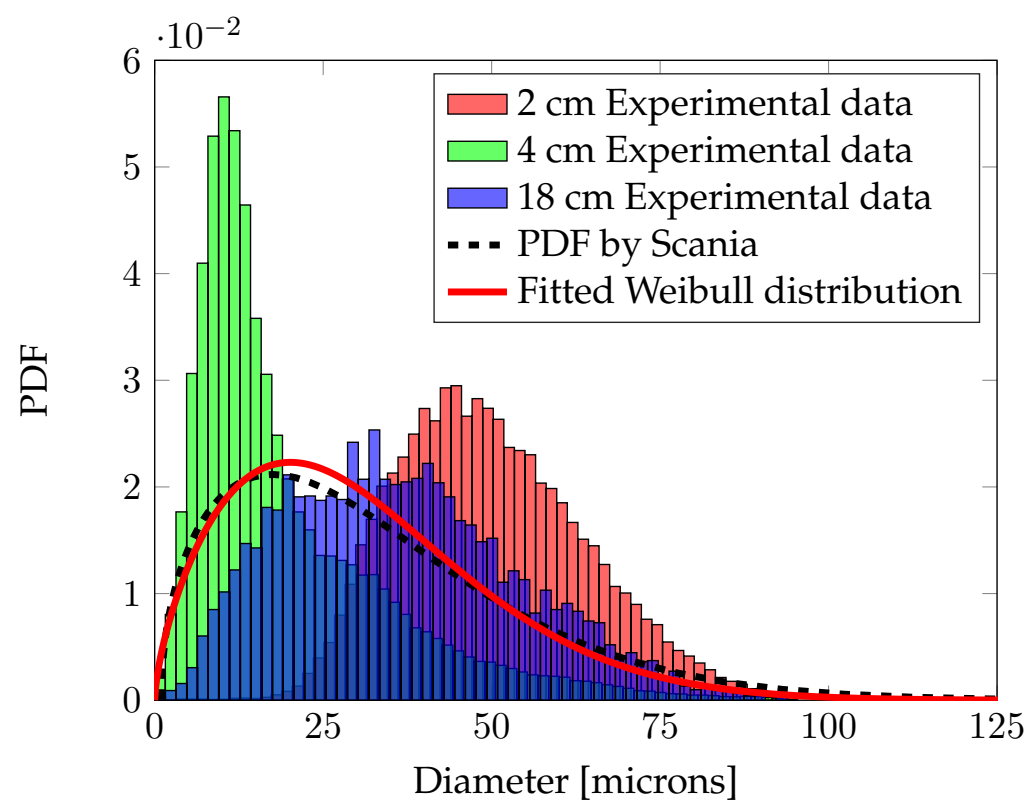

FIGURE 3.9: Probability Density Function for the droplet size distribution (Case 1a)

\subsubsection{Mass flow rate}

The gas phase (eulerian phase) characteristics in terms of velocity and turbulent intensity for mild and high cross-flows are displayed in figure 3.10 and 3.12. Flow separation in the diverging section of the chamber is noted in both cases and by studying the streamlines (see figure 3.11) a circulation motion is noticed. In the separation region the turbulent kinetic energy (intensity) increases due to a production of turbulence from the shearing of the mean flow. As a result most of the mass flow of the exhaust gas is limited to the upper region of the dosing chamber.
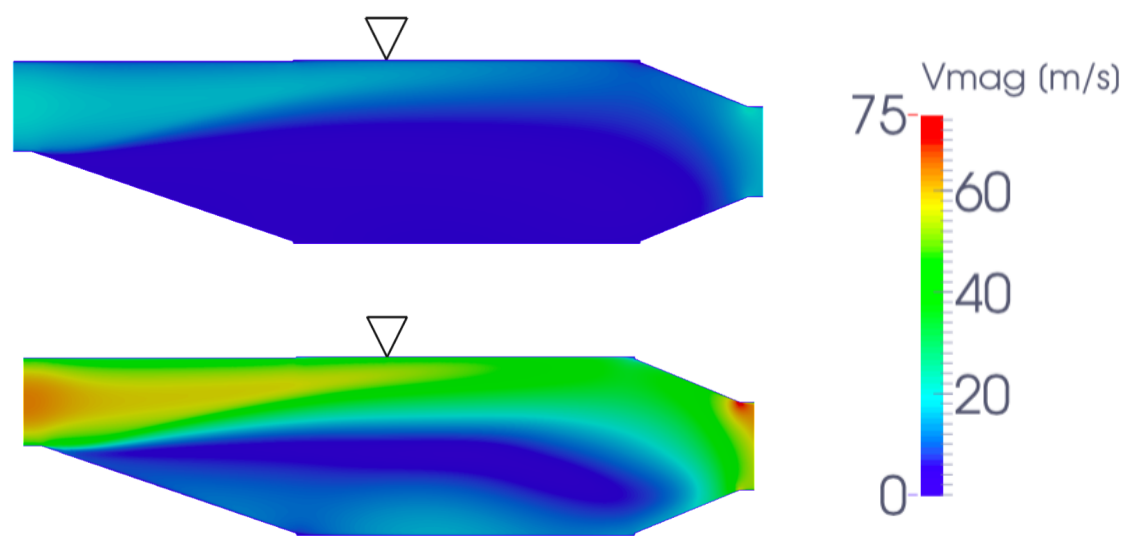

FIGURE 3.10: Velocity magnitude contours for mild crossflow $(400 \mathrm{~kg} / \mathrm{h})$ (above) and high cross-flow $(1400 \mathrm{~kg} / \mathrm{h}$ ) (below), $300{ }^{\circ} \mathrm{C}$ (Case $\left.2 \mathrm{~b} \& 4 \mathrm{~b}\right)$ 


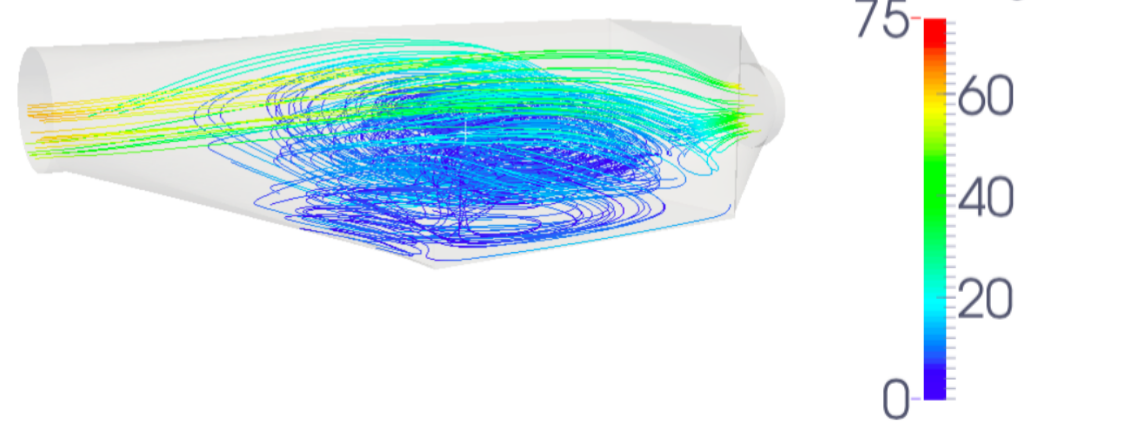

FIGURE 3.11: Streamlines colored by velocity magnitude for high cross-flow, $300{ }^{\circ} \mathrm{C}$ (Case $4 \mathrm{~b}$ )

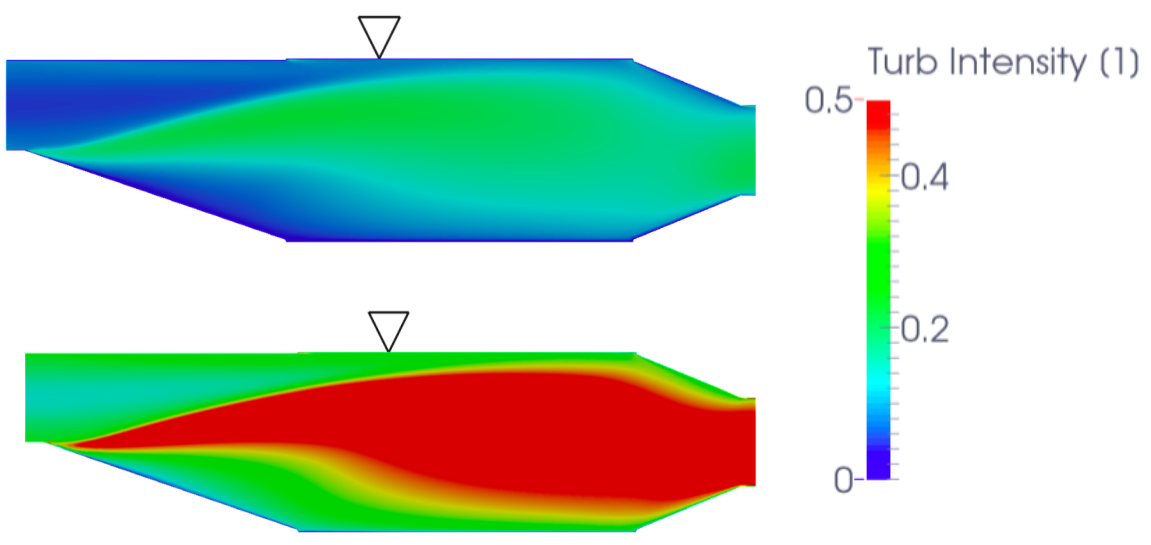

FIGURE 3.12: Turbulent intensity for mild cross-flow (above) and high cross-flow (below) based on mean velocity magnitude calculated as $15.4 \mathrm{~m} / \mathrm{s}$ at the inlet of mild cross-flow case, $300{ }^{\circ} \mathrm{C}$ (Case $2 \mathrm{~b} \& 4 \mathrm{~b}$ )

In figure $3.13,3.14$ and 3.15 the spray characteristics downstream in terms of size, velocity and mass flow rate are displayed. The size and velocity distributions are sampled in the vertical direction along the center lines as described in figure 3.8 and the y-axis shows the vertical position. It is noted that the penetration length is twice as large for the mild crossflow case compared to the high cross-flow case. It is also seen that the mild cross-flow case in general provides smaller droplet size distributions (figure 3.13). The "crook" in the size distribution at $Z / H \approx 0.6$ may be due to the transition of the gas flow between the high velocity region and the circulation region. This transition layer inherits a large velocity gradient which will accelerate breakup of droplets (higher Weber numbers). In terms of velocity droplets travelling close to the lower part of the domain inherit a negative streamwise velocity component meaning that they are trapped by the circulating flow and travelling backwards. As the mild cross-flow case inherits a smaller Weber number it is likely that the smaller droplets sizes are solely an effect of longer residence times and therefore more evaporation. This is further justified by studying the mass flow rates and residence times in figure 3.15 


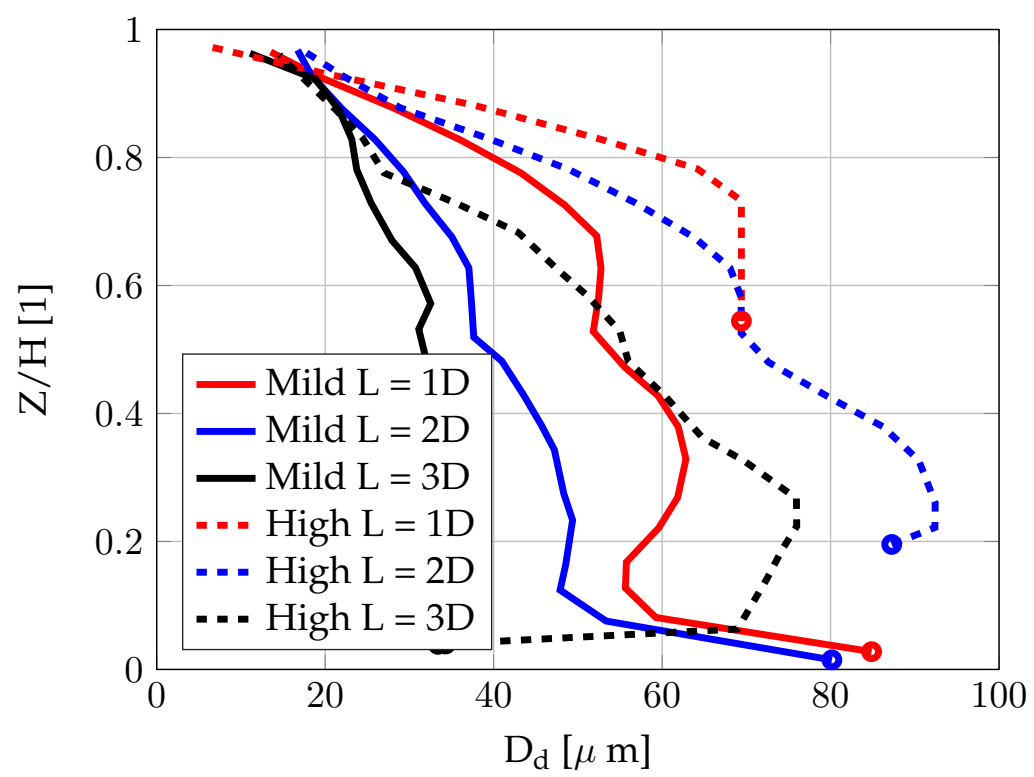

FIGURE 3.13: Vertical diameter distribution at a downstream distance of 1D, 2D and 3D (Case $2 b \& 4 b$ )

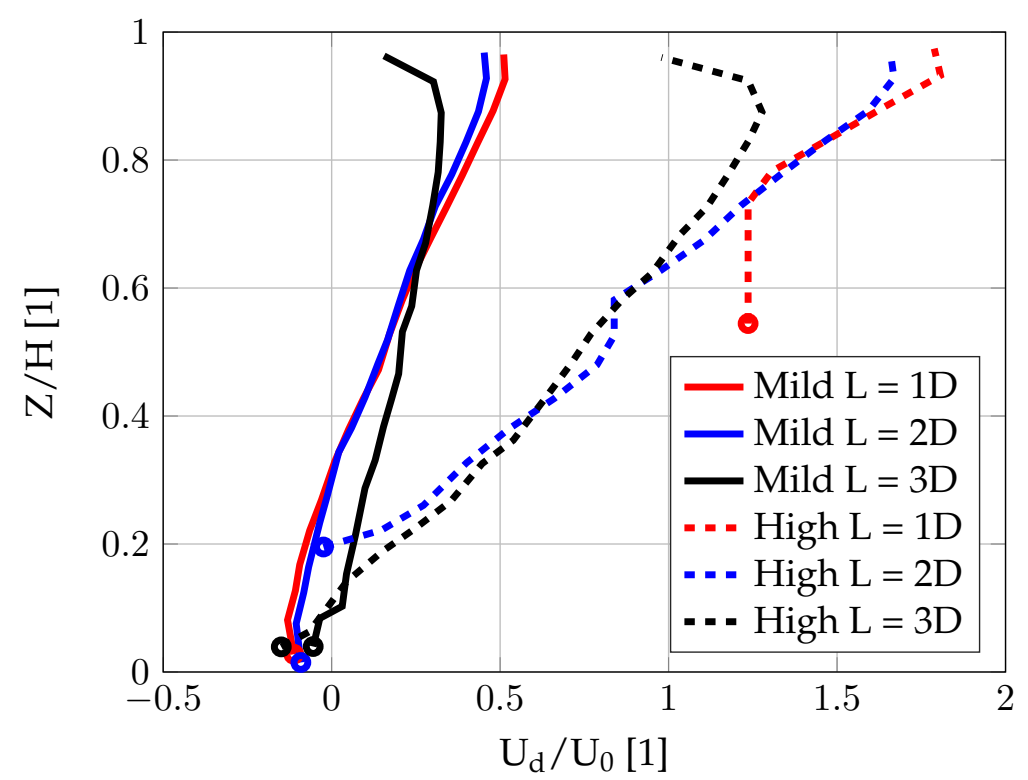

FIGURE 3.14: Vertical velocity distribution at a downstream distance of 1D, 2D and 3D (Case 2b \& 4b) 


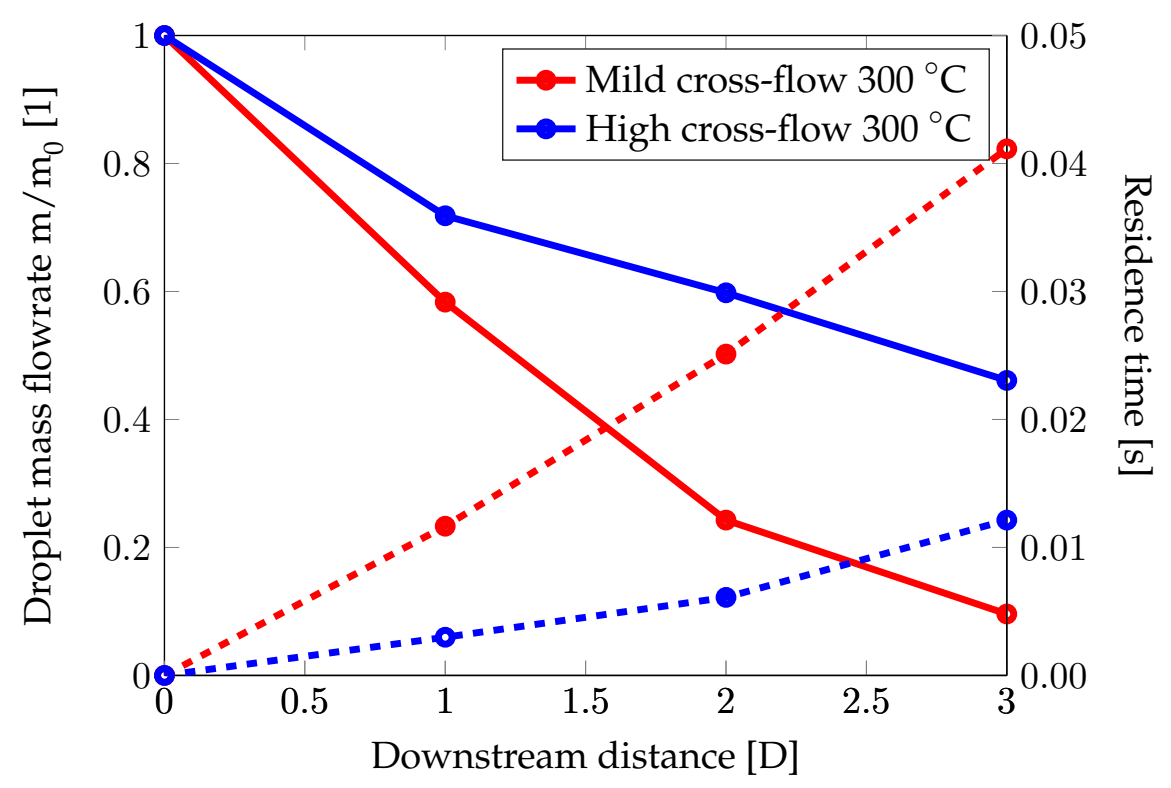

FIGURE 3.15: Spray mass flow rate downstream (Case 2b \& $4 b)$

\subsubsection{Temperature}

Figure 3.16, 3.17 and 3.18 displays the temperature sensitivity for constant mass flow rate of $1400 \mathrm{~kg} / \mathrm{h}$ (corresponding to the high cross-flow case). Despite a constant mass flow rate the velocity field for the eulerian phase (gas phase) changes due to varying density for the exhaust gas (assuming air in throughout this work). This can be seen by studying the penetration length in figure 3.16 as well as the gas-phase velocity in figure 3.17. The droplet mass flow rates for the different temperatures (figure 3.18) are very similar. This means that the increased evaporation due to higher temperatures is counter-affected by the increased gas velocity which gives smaller residence times (which will decrease evaporation). 


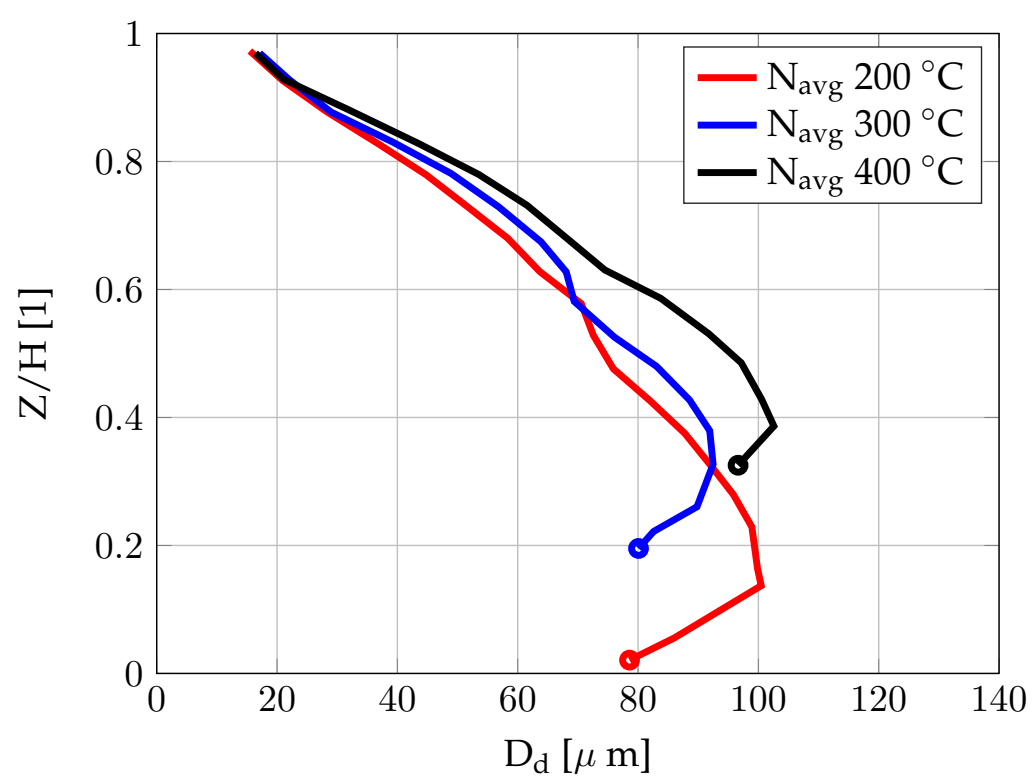

FIGURE 3.16: Vertical diameter distribution at a downstream distance of 1D, 2D and 3D (Case 4a, 4b \& 4c)

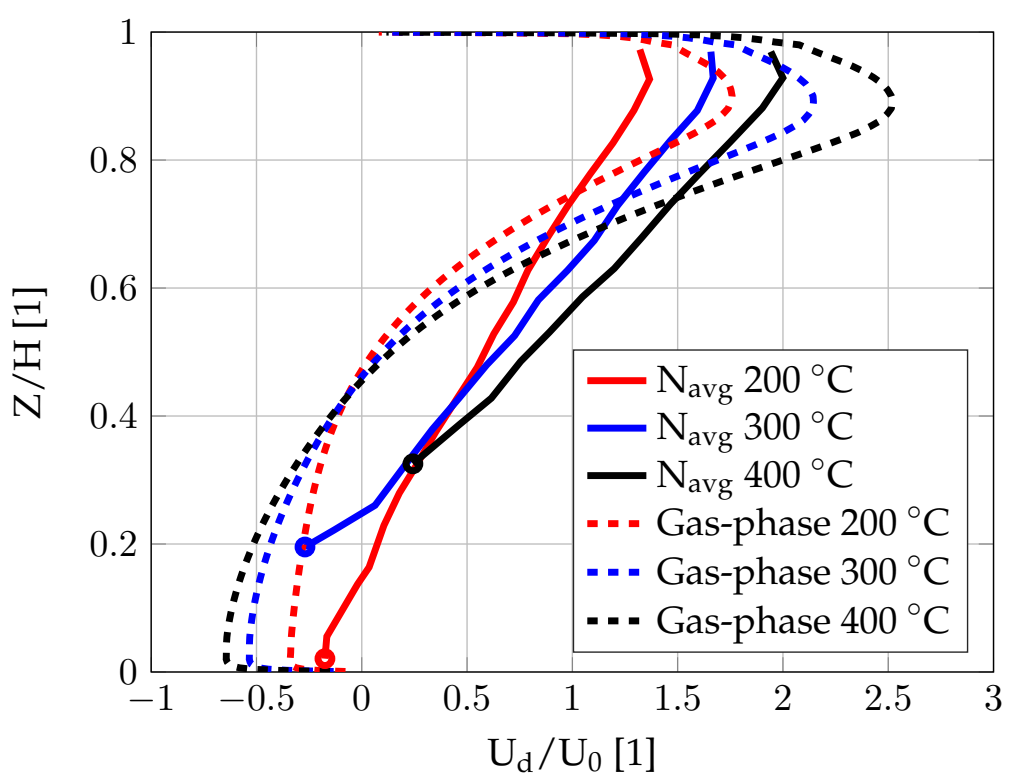

FIGURE 3.17: Vertical velocity distribution at a downstream distance of 1D, 2D and 3D (Case 4a, 4b \& 4c) 


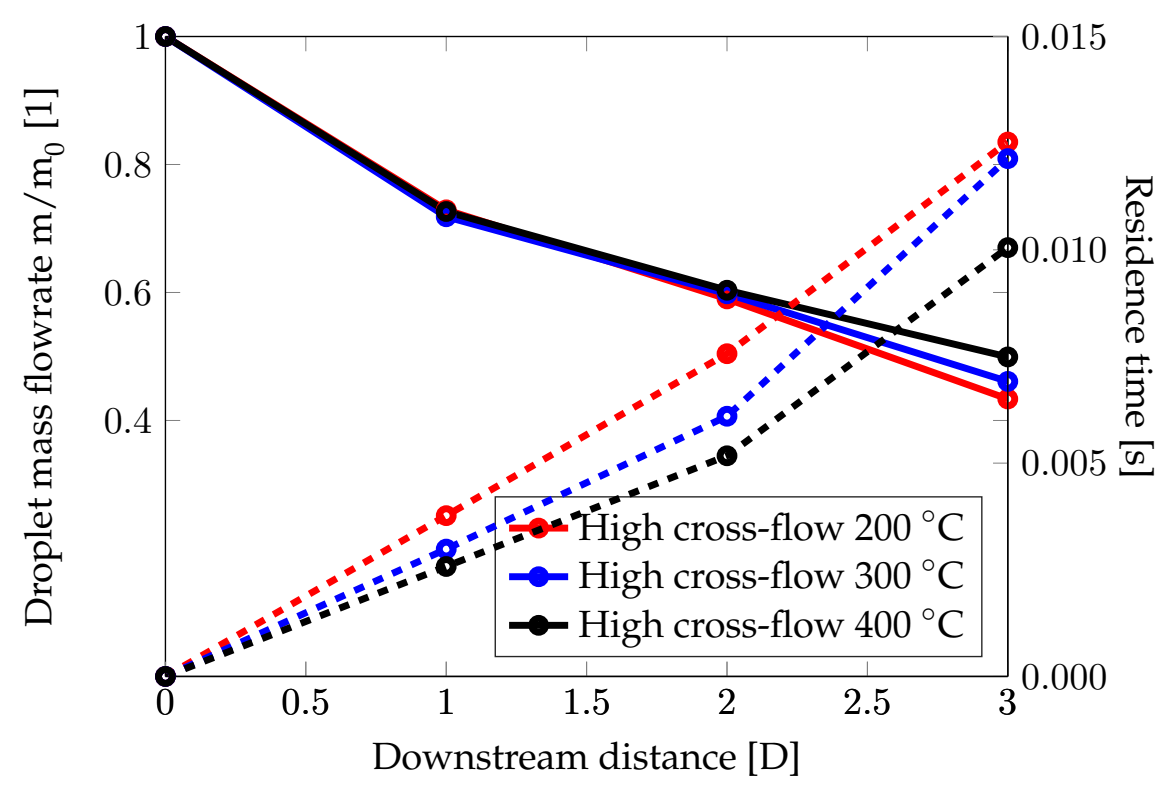

FIGURE 3.18: Spray mass flow rate downstream (Case 4a, $4 \mathrm{~b} \& 4 \mathrm{c})$

\subsubsection{Parcel sensitivity}

The concept of parcels as described per section 2.1.5 affects the preciseness of the simulation. Obviously with a lower number of parcels per unit time each parcel will need to statistically represent a larger number of discrete droplets. This can give erroneous results and it is therefore of interest to understand how the number of parcels affect the solution. In this section two different number of parcels per unit second are compared for the high cross flow case. It is noted that 200000 parcels/s (P200) seems appropriate since the use of 1000000 parcels/s (P1000 ) does not affect the size and velocity distributions significantly (figure 3.19 and 3.20). The evaporation rates (figure 3.21) are also close. 


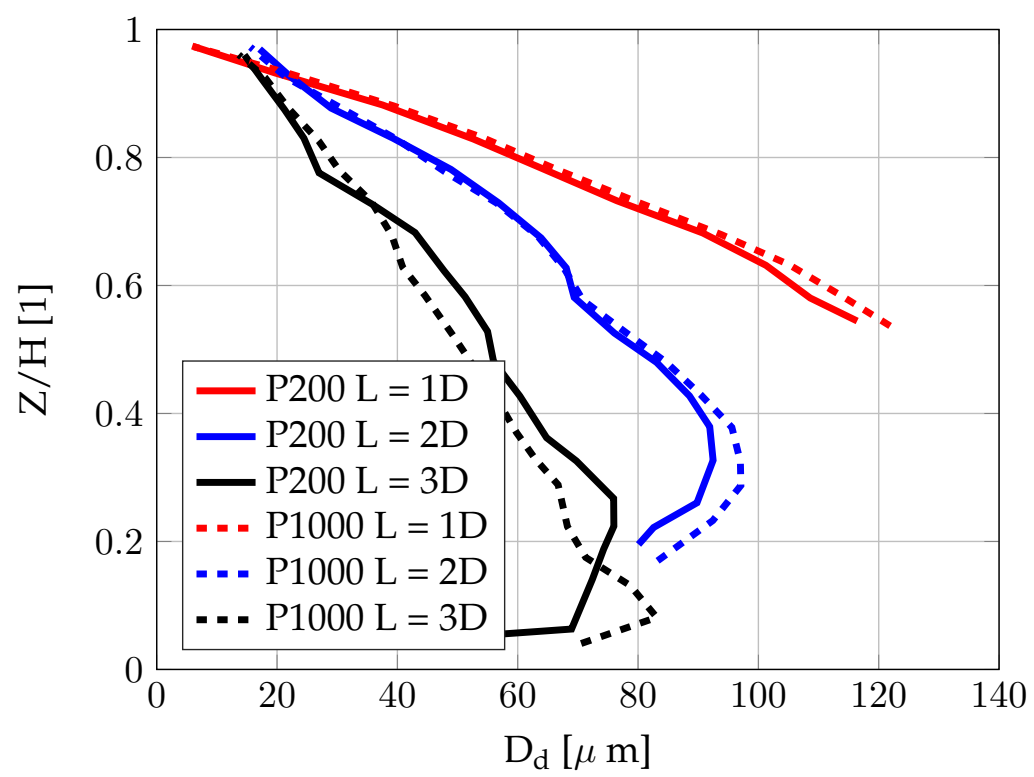

FIGURE 3.19: Vertical diameter distribution at $x=1 D$, 2D, 3D. $\mathrm{P} 200=200000$ parcels $/ \mathrm{s}$ and P1000 = 1000000 parcels/s (Case 4b \& 4b_s)

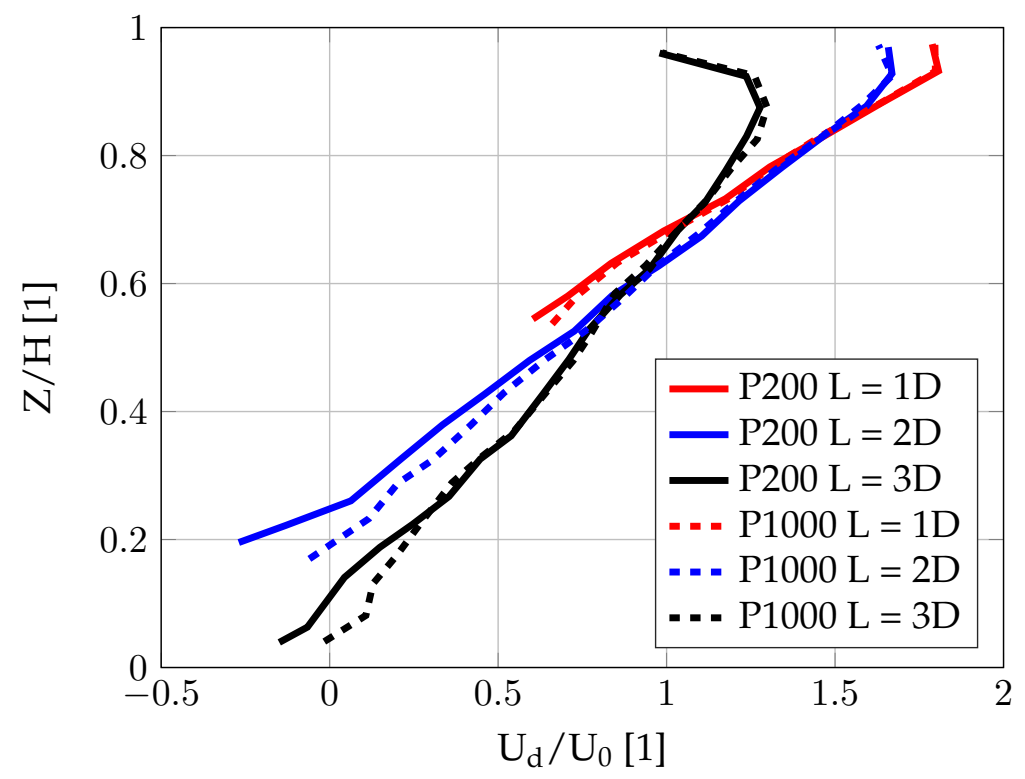

FIGURE 3.20: Vertical velocity distribution at $x=1 D$, 2D, 3D. $\mathrm{P} 200=200000$ parcels $/ \mathrm{s}$ and P1000 = 1000000 parcels/s (Case 4b \& 4b_s) 


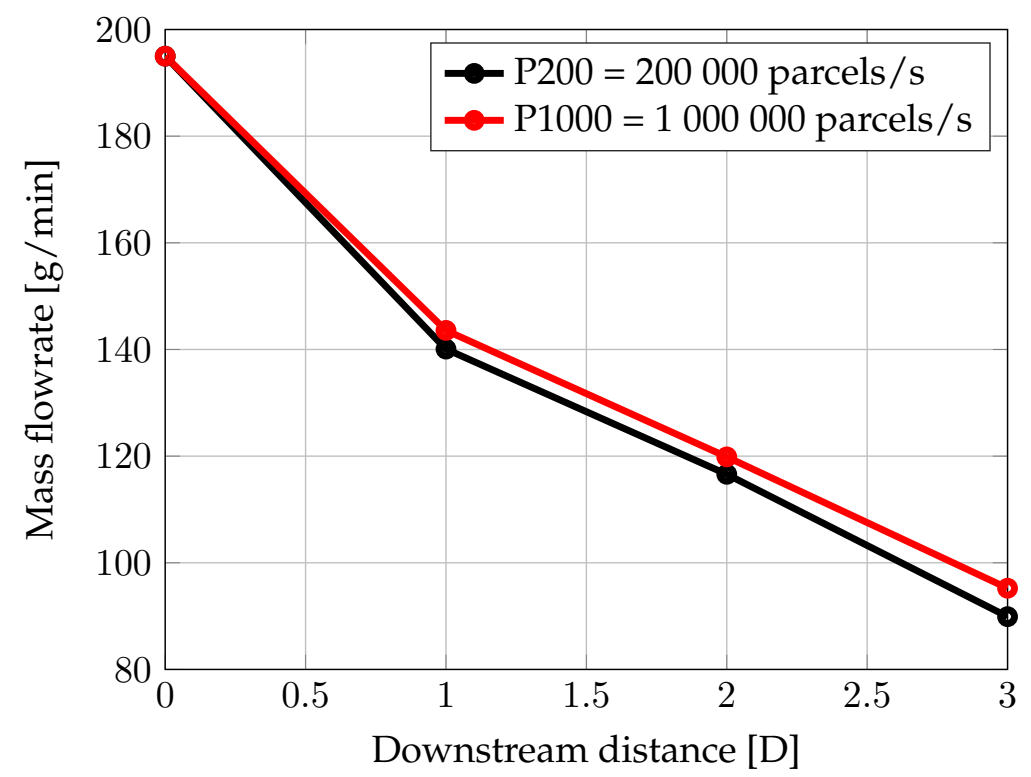

FIGURE 3.21: Spray mass flow rate downstream, P200 $=200$ 000 parcels $/ \mathrm{s}$ and P1000 $=1000000$ parcels $/ \mathrm{s}$ (Case $4 \mathrm{~b} \&$ $\left.4 \mathrm{~b} \_s\right)$ 


\section{Chapter 4}

\section{Conclusion}

The DEF spray evaporation and mixing inside an Urea-SCR dosing chamber has been numerically investigated. The numerical setup was based on a preliminary study of the numerical framework in terms of a sub-model sensitivity analysis. The conclusions from the sub-model sensitivity analysis is presented in section 3.1.4 and involves recommendations for turbulence coupling, evaporation model and droplet breakup model.

The results from the Urea-SCR in terms of evaporation and mixing of the spray may lead to several conclusions. First evaporation rates seem to be higher for the mild cross flow due to longer residence times. However when it comes to temperature the evaporation rates appears unaffected as a higher temperature is countered by lower residence time due to compressibility of the exhaust gas. Further it can be concluded that higher gas velocities gives lower spray penetrations lengths. Finally a sensitivity analysis on the number of parcels was conducted and it appears that 200000 parcels/s capture the spray dynamics properly.

This study may be of interest as a basis for further work on numerical simulations of Urea-SCR. Since a turbulence model is used (Realizable $K-\epsilon$ ) in this work it could be of interest to perform LES simulations which eliminates the need of a turbulence coupling. This could potentially also be useful for studying the gas flow separation closer as this phenomena heavily affects the mixing of the DEF spray.

Another important finding in this study is the validation of injection properties. As noted in section 3.2.1 the size distribution given and used as injection properties show a very coarse correspondence to experimental results. This is probably due to the methodology around measuring size distribution as it is common to measure at a certain distance from the nozzle. In the future it is essential to keep a clear communication regarding size distributions as numerical simulations can only be as accurate (ideally) as the boundary conditions given.

Throughout this work many simulations have been carried out. Due to a limited amount of time results have been extracted as soon as stable conditions are assumed. As mentioned in section 2.3.2 stable conditions are assumed from visual inspection and an approximation of the number of droplet flow throughs derived from the residence times. It could be of interest to run longer simulations and study a larger number of flow quantities in order to make sure that stable conditions are reached. 



\section{Bibliography}

Apte, S. V., M. Gorokhovski, and P. Moin (2003). "LES of Atomizing Spray with Stochastic Modeling of Secondary Breakup". In: International Journal of Multiphase Flow 29, pp. 1503-1522.

Beale, J. C. and R. D. Reitz (1999). "Modeling Spray Atomization with the Kelvin-Helmholtz/Rayleigh-Taylor Hybrid Mode". In: Atomization and Sprays 9, pp. 623-650.

Ferrer, Mireia Altimira (2010). "Analysis of Atomization Systems through Mathematical Modeling and Experimentation: Application to Industrial Fan-Spray Atomizers". PhD thesis. Universidad De Navarra, Escuela Superior De Ingenieros, San Sebastian.

Gosman, A. D. and E. Ioannides (1983). "Aspects of computer simulation of liquid-fuelled combustors". In: J. Energy. 7, pp. 482-490.

Guide, Fluent Theory (2013). Ansys Fluent Theory Guide 15.0. ANSYS Inc.

Hart, Robert (2006). "Numerical modelling of tunnel fires and water mist suppression". PhD thesis. School of Chemical, Environmental and Mining Engineering, University of Nottingham.

Iannantuoni, Luca et al. (2013). "Validation and Assessment of a Water Mist Multi-hole Nozzle Model for Fire Simulations". In: Springer Science Fire Technology.

Issa, R.I. (1986). "Solution of Implicitly Discretized Fluid Flow Equations by Operator Splitting". In: Journal of Computational Physics 62, pp. 40-65.

Kader, B. (1981). "Temperature and Concentration Profiles in Fully Turbulent Boundary Layers". In: Int. J. Heat Mass Transfer 24, pp. 1541-1544.

Kuo, K. K. Y. (1986). Principles of Combustion. John Wiley and Sons, New York.

Levich, V. G. (1962). Physicochemical Hydrodynamics. Prentice Hall.

Liu, Zhigang and Andrew K. Kim (2000). A Review of Water Mist Fire suppression systems - Fundamental Studies. Tech. rep. National Research Council Canada.

Miller, R. S., K. Harstad, and J. Bellan (1998). "Evaluation of Equilibrium and Non-Equilibrium Evaporation Models for Many Droplet Gas-Liquid Flow Simulations". In: International Journal of Multiphase Flow 24(6), pp. 10251055.

Morsi, S. A. and A. J. Alexander (1972). "An Investigation of Particle Trajectories in Two-Phase Flow Systems". In: J. Fluid Mech. 55(2), pp. 193208.

Nova, I. and E. Tronconi (2014). Urea-SCR Technology for deNOx After Treatment of Diesel Exhausts. Springer Science.

O'Rourke, P. J. (1981). "Collective Drop Effects on Vaporizing Liquid Sprays". $\mathrm{PhD}$ thesis. Princeton University.

Patterson, M. A. and R. D. Reitz (1998). "Modeling the Effects of Fuel Spray Characteristics on Diesel Engine Combustion and Emission". In: SAE Paper 9. 
Prosperetti, Andrea and Gretar Tryggvarson (2007). Computational Methods for Multiphase flows. Cambridge University press.

Ranz, W. E. and W. R. Marshall (1952). "Vaporation from Drops, Part I". In: Chem. Eng. Prog 48(3), pp. 141-146.

Reitz, R. D. and F. V. Bracco (1986). Mechanisms of Breakup of Round Liquid Jets. ed. N. Cheremisnoff. The Encyclopedia of Fluid Mechanics, pp. 223-249.

Reitz, R.D. (1987). "Mechanisms of Atomization Processes in High-Pressure Vaporizing Sprays". In: Atomization and Spray Technology 3, pp. 309-337.

Sazhin, S. S. (2006). "Advanced Models of Fuel Droplet Heating and Evaporation". In: Progress in Energy and Combustion Science. Elsevier Science 32, pp. 162-214.

Shih, T.-H. et al. (1995). “A New - Eddy-Viscosity Model for High Reynolds Number Turbulent Flows - Model Development and Validation". In: Computers Fluids.

Tanner, F.X. (2011). Handbook of Atomization and Sprays. Springer Science.

Taylor, G. I. (1963). The Shape and Acceleration of a Drop in a High Speed Air Stream, Technical Report. Tech. rep. Scientific Papers of G. I. Taylor. ed., G. K. Batchelor.

Tropea, Cameron, Alexander L. Yarin, and John F. Foss (2007). Handbook of Experimental Fluid Mechanics. Springer-Verlag Berlin Heidelberg. 